\title{
Discrimination and behavioral responses to communication signals compared across Apteronotids.
}

\author{
Danielle Leigh Dillon-Seeger \\ dldillonseeger@mix.wvu.edu
}

Follow this and additional works at: https://researchrepository.wvu.edu/etd

Part of the Behavioral Neurobiology Commons, and the Other Neuroscience and Neurobiology

\section{Commons}

\section{Recommended Citation}

Dillon-Seeger, Danielle Leigh, "Discrimination and behavioral responses to communication signals compared across Apteronotids." (2019). Graduate Theses, Dissertations, and Problem Reports. 7392. https://researchrepository.wvu.edu/etd/7392

This Thesis is protected by copyright and/or related rights. It has been brought to you by the The Research Repository @ WVU with permission from the rights-holder(s). You are free to use this Thesis in any way that is permitted by the copyright and related rights legislation that applies to your use. For other uses you must obtain permission from the rights-holder(s) directly, unless additional rights are indicated by a Creative Commons license in the record and/ or on the work itself. This Thesis has been accepted for inclusion in WVU Graduate Theses, Dissertations, and Problem Reports collection by an authorized administrator of The Research Repository @ WVU. For more information, please contact researchrepository@mail.wvu.edu. 


\section{Discrimination and behavioral responses to communication signals compared across Apteronotids.}

\section{Danielle L. Dillon-Seeger}

Thesis submitted to the Eberly College of Arts and Sciences at West Virginia University in partial fulfillment of the requirements for the degree of

\section{Master of Science in Biology}

Gary Marsat, Ph.D., Chair

Clifton Bishop, Ph.D.

Sadie Bergeron, Ph.D.

Department of Biology

\section{Morgantown, West Virginia}

\section{9}

Keywords: Chirps, Communication, Behavior, Electroreception, Perception. Copyright 2019 Danielle L. Dillon-Seeger 


\begin{abstract}
Discrimination and behavioral responses to communication signals compared across Apteronotids.
\end{abstract}

\title{
Danielle L. Dillon-Seeger
}

Sensory systems are often uniquely tailored to encode behaviorally relevant signals and comparative studies across species can thus reveal how evolutionary changes shape sensory functions. The structure of communication signals varies widely between ghost knifefish species. Recent findings suggest that the nervous system co-adapted to the various signal structures observed across species to support different sensory behaviors. The aim for this thesis was to compare the sensory behavior of 3 species of ghost knifefish to contrast their behavioral performance with the known differences in neurophysiology. We hypothesize that for the different signal types and species, the ability to discriminate small variations in chirp properties will match the neural encoding method and accuracy employed in the sensory system. We used a habituation-dishabituation paradigm to reveal the ability to discriminate chirp variants. We found that in two species discrimination ability depended on the frequency of the background beat signal and thus on context. For low-frequency beat typical of same-sex interactions, A. albifrons discriminated chirp variants but not A. leptorhynchus but the contrary was observed for high frequency beats characteristic of male-female interactions. A third species, A. devenanzii accurately discriminated all chirp signal independently of the frequency of the background beat. These differences match the differences in sensory encoding previously characterized. We argue that these results thus establish a clear link between signal structure, neural coding strategy and perceptual tasks. These new findings provide an important step forward in our understanding of the co-evolution sender and receiver mechanisms. 


\section{Acknowledgments}

This is just a short list of people and entities that have enabled me to further my education and experiences. If it were possible to include everyone that has helped me through the years it would be pages long. There may even be a few people I failed to mention, however, I am nonetheless grateful.

First of all, I would like to express my gratitude to WVU for granting me this opportunity and all of the support. I would also like to thank Katrina Stewart, Cheryl Walton, Christina ByrneHoffman, and Sophia Zhang. I learned so much teaching BIOL 116 and 118 through the years. Your never ceasing support and encouragement was (and still is) greatly appreciated. I leave with a new found appreciation for teaching academics and along the way came to realize that I enjoy teaching.

My sincere thanks go to my committee members, Drs. Bishop and Bergeron for your time, advice and support. Dr. Bishop (or Cliff as you preferred to be addressed) thank you so much for all of the advice and encouragement through the years. You were there since day one and helped me through some difficult times.

Dr. Marsat, I cannot thank you enough for all that you have done. Your kindness, patience, and willingness to not only guide, but also help was more than I could ask for. I greatly admire and appreciate you.

Thank you to my fellow lab mates: Kathryne Allen, Sree Montipally, Keshav Ramachandra, and Oak Milam. Spending time with you all (and Dr. Marsat) in and out of lab was so much fun. We laughed till we cried, cried at times, and even took turns picking on each other (which I usually started). It was all a wonderful experience. A special thanks goes to Keshav and Oak for putting up with my antics and Kate for always joining in.

I would also like to thank the undergraduates that helped me collect and analyze data. A special thanks goes to Ian McCoy and Robin Oliverio. You were there from the start and saw me through to the end. I really enjoyed our time together and learned much from all of you. Your willingness to go the extra mile and help out did not go unnoticed.

I cannot finish without thanking my family, without them I would not be here. Mom and dad, thank you for being there for me through good times and bad. To my brothers, Brandon and Jordan, thank you for the encouragement and always being there. To my husband, Steven, thank you for the continued support through the years. 


\section{Table of Contents}

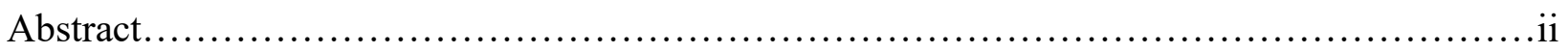

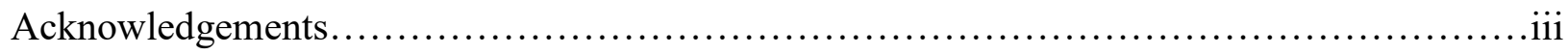

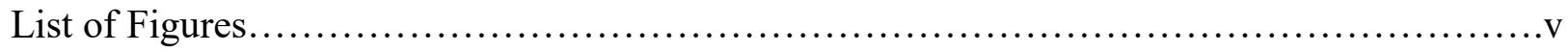

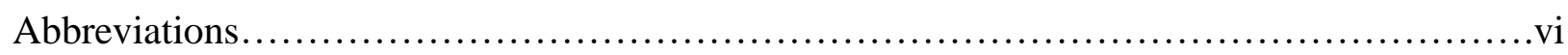

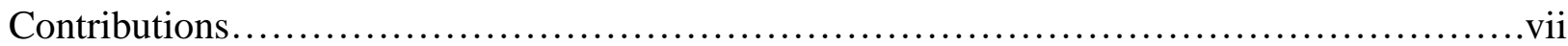

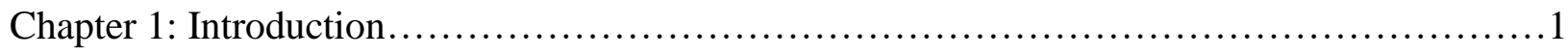

I. Sender-Receiver Matching ......................................

II. Communication Behavior and Evolution................................

III. Communication Signals in Ghost Knifefish .........................5

IV. Species-Specific Aspect of Electrocommunication.....................

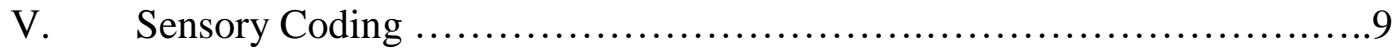

Chapter 2: Methods........................................................... 14

Chapter 3: Results........................................................... 19

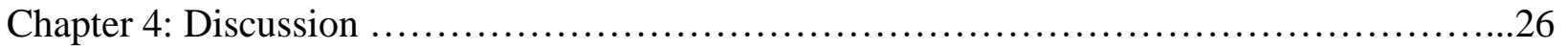

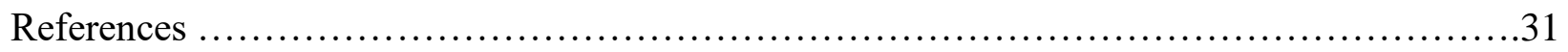




\section{List of Figures}

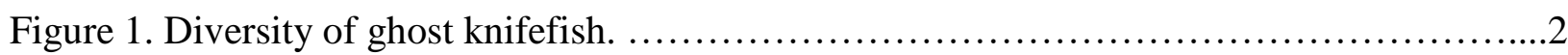

Figure 2. Properties of communication signals in 3 ghost knifefish...........................9

Figure 3. Neurophysiological responses of A. leptorhynchus ELL neurons showing that chirps on HFBs could be discriminated while responses for LFBs do not encode chirp properties...........10

Figure 4. Neurophysiological responses of A. albifrons ELL neurons showing that chirps on LFBs could be discriminated while responses for HFBs do not encode chirp properties..........11

Figure 5. Neurophysiological responses of $A$. devenanzii ELL neurons showing the accurate discrimination of chirps for both LFBs and HFBs.........................................12

Figure 6. Habituation-Dishabituation testing set-up and analysis........................... 15

Table 1. Stimuli Sets for A. devenanzii, A. albifrons, and A. leptorhynchus ......................17

Figure 7 Custom tracking program. ................................................... 18

Figure 8. A. leptorhynchus responses to chirp on HFBs or LFBs measured over the 1 min of each

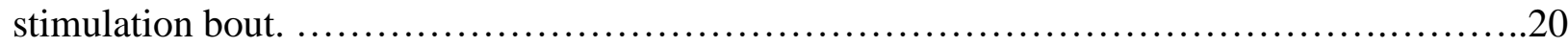

Figure 9. A. leptorhynchus shows strong dishabituation for HFBs chirp stimuli when considering

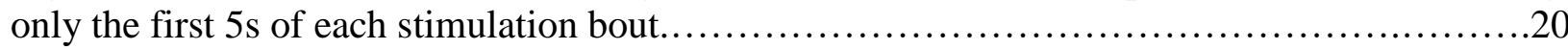

Figure 10. A. leptorhynchus responses displayed a dishabituation effect only if the fish is far

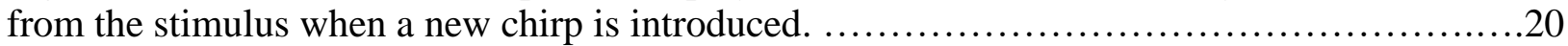

Figure 11. A. albifrons responses to chirp on HFBs or LFBs measured over the 1 min of each

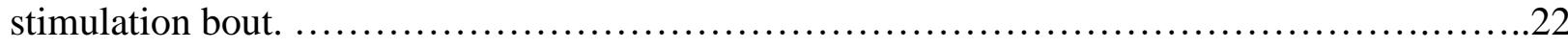

Figure 12. A. albifrons shows dishabituation for LFBs chirp stimuli when considering only the

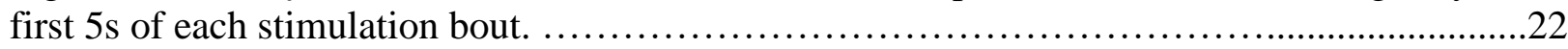

Figure 13. A. albifrons shows an increase in swimming speed for LFB after chirp change regardless of the position of the fish at the start of the stimulus. .............................22

Figure 14. A. devenanzii responses to chirp on HFBs or LFBs measured over the $1 \mathrm{~min}$ of each

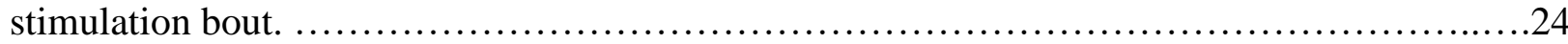

Figure $15 \mathrm{~A}$. devenanzii response during the first $5 \mathrm{~s}$ of stimulus show dishabituation for all chirp stimuli when considering the first $5 \mathrm{~s}$ of each stimulation bout................................. 24

Figure 16 A. devenanzii shows dishabituation in position not speed regardless of stimulus type or

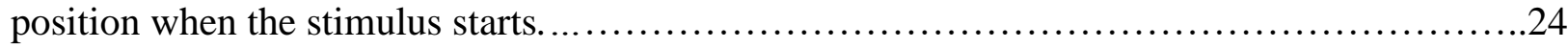




\section{Abbreviations}

$\begin{array}{ll}\text { AFR } & \text { Abrupt Frequency Rise } \\ \text { AM } & \text { Amplitude Modulation } \\ \text { ELL } & \text { Electrosensory Lateral Line Lobe } \\ \text { DF } & \text { Difference Frequency } \\ \text { EOD } & \text { Electric Organ of Discharge } \\ \text { FM } & \text { Frequency Modulation } \\ \text { GFR } & \text { Gradual Frequency Rise } \\ \text { HFB } & \text { High Frequency Beat } \\ \text { JAR } & \text { Jamming Avoidance Response } \\ \text { LFB } & \text { Low Frequency Beat } \\ \text { PC } & \text { Pyramidal Cells }\end{array}$




\section{Contributions}

The results presented in this thesis were a collaborative effort in the Marsat lab. The experimental design was determined by Danielle Dillon-Seeger (DDS) and Dr. Gary Marsat. The behavioral experiments were run by DDS with the aid of several undergraduate assistants (including Ian McCoy and Robin Oliverio). The analysis and tracking programs were created by Dr. Marsat and Keshav Ramachandra. The analysis was performed by DDS with the aid of the undergraduate assistants and Dr. Marsat. The experiments and analysis were orchestrated by DDS and the project, as a whole, was managed by DDS under Dr. Marsat's supervision. A small portion of the dataset for A. leptorhynchus responses was used in a previous publication (Allen and Marsat, 2018) but the movement data was re-analyzed for this manuscript and are presented in the results section. 


\section{Chapter 1: Introduction}

\section{Sender-Receiver Matching}

Sensory systems must not only detect a signal but must also be capable of decoding this signal into meaningful information that the animal can use. The output of this processed information can often be seen and tested as a resulting behavior. The sender-receiver matching hypothesis has been studied across many modalities and model organisms and is particularly relevant to animal communication behavior. The co-evolution of the sender's signal and the physiology of the receiver's sensory system is expected to closely match in properties (Guilford and Dawkins, 1993; Gall et al., 2012). How signal structure and sensory processing mechanisms are adapted to mediate behavior is of particular interest in neuroscience. The detection and discrimination of communication signals via behavior has been particularly well studied across many species. This is possible because the sending and receiving mechanisms evolve in the same organisms and are shaped by the pressure of having efficient interactions with conspecifics. For example, during courtship, many singing anurans (Wilczynski et al., 1993; Wollerman and Wiley, 2002), insects (Wells and Henry, 1992), and birds (Woolley and Moore, 2011; Gall et al., 2012; Baker et al., 2016) show preference for specific song characteristics but most importantly, their auditory tuning matches these properties. Across species there is evidence of sender receiver matching in signal features such as pulse duration (Kostarakos and Hedwig, 2012), frequency (Ryan and Wilczynski, 2019), and rise time (Gall et al., 2012). These are a few examples that stress that it is advantageous for the sender and receiver to adapt and co-evolve in a complementary manner so that signal and sensory system can efficiently encode and extract important information. 


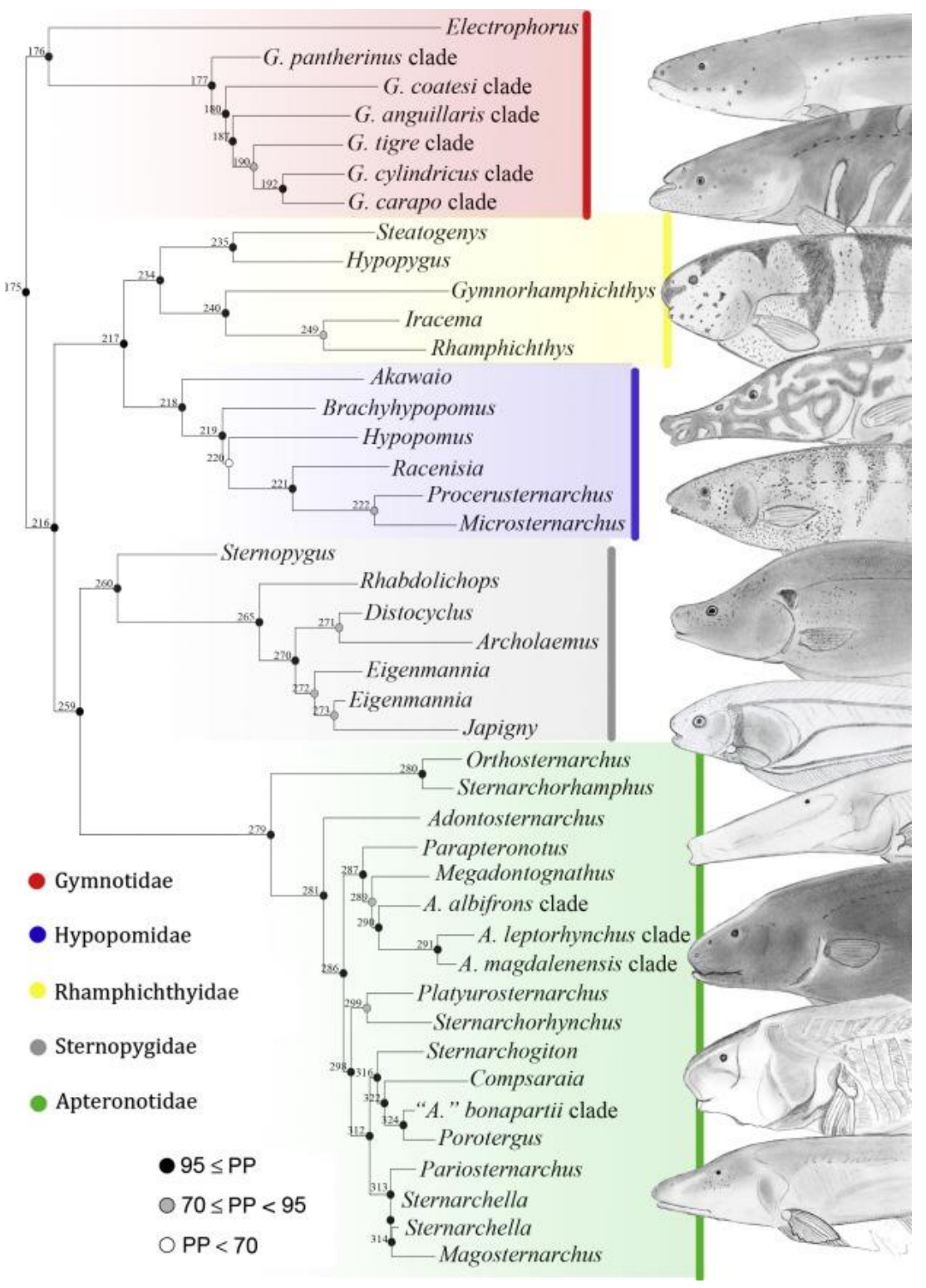

Figure 1. Diversity of ghost knifefish. Modified from (Tagliacollo et al., 2016). The three species of interest in this study are represented in Apteronotidae (green), Apteronotus leptorhynchus, Apteronotus albifrons and Adontosternachus (specifically we use A. devenanzii).

The evolution of communication has been well studied in weakly electric, South American knifefish, including the Apteronotidae family (a.k.a. Apteronotids or ghost knifefish; Fig. 1). This large and species-rich family displays countless variations in size, body morphology, sexual dimorphism, and communication signals (Alves-Gomes et al., 1995; Compton and Albert, 2006; Turner et al., 2007). Due to a rich existing literature detailing the sensory mechanisms and the behaviors in these fish, they are a powerful model system in 
neuroscience and the cross-species variability makes it a good target for comparative studies (Allen and Marsat, 2018, 2019; Tallarovic \& Zakon, 2005; Turner et al., 2007). We know that the basic tuning properties of electroreceptors match the frequency of the species-specific signals across ghost knifefish (Hopkins, 1976). However, as in most other model systems, the senderreceiver matching principles has only been revealed for basic signal properties (e.g. carrier frequency) and peripheral neuron's tuning. It is unclear whether this concept can explain variations in neural properties in higher brain areas by presenting a match between neural coding strategies and more complex aspects of the signals. In this thesis, we aim to contribute to recent advances in our understanding of sensory processing in knifefish by describing how perceptual abilities vary across species. By relating the perceptual abilities determined here to the recently characterized sensory processing strategies and the well-known signal structures, we will determine how the co-evolution of signals and sensory systems support specific perceptual abilities that mediate behavior.

\section{Communication Behavior and Evolution}

Communication signals are particularly well suited to examine the coevolution of sender and receiver mechanism and to understand how sensory mechanisms adapt to efficiently capture relevant aspects of the signals. Communication behaviors typically arise from either a sender precursor or a receiver precursor (Bradbury and Vehrencamp, 2011). In the former, a preexisting mechanism that can be used to send a signal carries some information about a behaviorally relevant condition (e.g. fitness of the sender). The receiver then evolves the mechanism to receive and discriminate the signal. Given a reliable correlation between the signal and the condition, evolutionary pressure can improve both sending and receiving mechanisms if the signal is beneficial for both sender and receiver. The result is typically a highly stereotyped and efficient communication behavior. A good example of this phenomenon is bioluminescence in fireflies that is thought to have first evolved to fence of predators but then became a signal to attract mates (Buck and Buck, 1966; Bradbury and Vehrencamp, 2011). A communication behavior can also arise from a receiver precursor were a sensory sensitivity precedes the evolution of a signal using this sensory channel. For example, in some populations of guppies, sensory sensitivity and attraction towards orange items is thought to have initially evolved to 
locate a specific food source rich in beta-carotene (i.e. certain fruits that fall in the water in these regions). Males then evolves patches of orange color on their body to attract females and the preexisting attraction to this color made it an effective channel (Rodd et al., 2002). In weakly electric fish the ability to sense electric field arose first as a way to detect other organisms and the ability to generate electric field came after (Alves-Gomes, 2001).

To be beneficial to both sender and receiver, communication signals must carry reliable information about a condition. To ensure this reliability, signals typically have a built-in honesty guarantee. Four categories of guarantees have been delineated: 1) Handicap signals where the honesty of the signal is guaranteed by the cost of bearing the signal (e.g. peacock tail) (Loyau et al., 2005). 2) Index signals that can only be produced if certain conditions are true (high claw marks on a tree signal the territory of a large tiger) (Smith et al., 1989; Bradbury and Vehrencamp, 2011). 3) Signals with a proximity cost where the cost of deception is being called on the bluff and suffering the physical consequences (e.g. deceitfully signaling the motivation to attack) (Dawkins and Guilford, 1991; Hasson, 1994). 4) Conventional signal can exist without honesty guarantee when the interest of the sender and receiver are aligned, otherwise honesty is tested behaviorally by the receiver (similar to signals with a proximity cost). Signals that are costly to produce are particularly common in aggressive and courtship context because they attest to the fitness of the individual (Clutton-Brock and Albon, 1979). Loud vocal calls that require energy to produce, complex nest decorations that take time to build or elaborate dances that require vigorous and agile movements are some of the examples that are well known (Bradbury and Vehrencamp, 2011). Several hypotheses regarding the honesty of communication signals in knifefish can be emitted. In species with sexual dimorphism in the EOD signal, sex hormones influence the frequency of the EOD and of the chirps which can thus serve as a reliable indicator of physiological state (Engler and Zupanc, 2001; Cuddy et al., 2012). Increasing the frequency of the EOD beyond their baseline level is costly because it requires a higher discharge rate (Salazar et al., 2013). It is thought to be for this reason that chirp that have a high frequency rise are accompanied by an amplitude decrease: it is hard to increase frequency and maintain the amplitude of the output. Some aspect of these communication signals might reflect an established convention. For example, the pattern of chirp exchange might be informative (see below) but has no obvious cost. 
Various aspect of signals can be correlated with a condition. The relevant variable (color, song pattern, odor blend) can exist along a continuum or be categorical. Several examples illustrate this contrast well and often exist within the same species. In Towhee birds the first part of the call serves to attract the attention and does carry information in graded variation of the sound element (Richards, 1981). The second part of the call however varies along a continuum

and is evaluated by mates and competitors. In meerkats, distinct alarm calls are emitted for aerial and terrestrial predators and the responses are categorically different (Moran, 1984; Bradbury and Vehrencamp, 2011). However, these same calls can vary along another dimension indicating the urgency of the threat. For discreet vs graded signals, the sensory system is faced with two different tasks: detection vs discrimination. Neurophysiological finding in ghost knifefish suggest that some types of chirps are perceived categorically without distinguishing between variations in chirps' structure whereas for other chirps, details of the chirps are encoded by the nervous system and could influence behavior (see section IV below).

\section{Communication Signals in Ghost Knifefish}

Ghost knifefish produce a continuous oscillating electric organ discharge (EOD) (Hopkins, 1988; Zakon and Smith, 2010; Zupanc et al., 2006), which they use to navigate their environment and communicate with conspecifics. EODs can relay important information, such as age, breeding status, sex, and rank. When two or more weakly electric fish are relatively close to one another, their EODs interact. Constructive or destructive interactions between the two EOD, depending on their relative phase, lead the combined signal to have cyclical amplitude modulations (AM). This particular type of AM is known as a beat. Since many species are sexually dimorphic in their EOD frequency ranges, large differences in EOD frequencies lead to high beat frequencies, or high frequency beats (HFBs), during male-female interactions. Low beat frequencies, or low frequency beats (LFBs), occur in same sex interactions in these dimorphic species.

All species transiently modulate or increase their EOD frequency in a variety of social interactions (Hagedorn and Heiligenberg, 1985; Bastian and Nguyenkim, 2001; Zakon et al., 2002; Hupé and Lewis, 2008; Zakon and Smith, 2010). These signals are classified in several 
categories: gradual or abrupt frequency rises (GFR or AFR), jamming avoidance responses (JAR) or chirps.

GFRs are characterized as a smooth increase in EOD frequency and a gradual decrease back to the baseline. GFRs last from tens of milliseconds to several minutes (Zakon et al., 2002; Zupanc et al., 2006) making them longer in duration than chirps, but less conspicuous. There is disaccord in categorizing rises or how many types exist. According to Hagedorn and Heiligenberg there are two types, short (1s duration) and long (tens of seconds) rises (1985). In contrast Engler et al. (2000) believed that all rises fell along a continuum. Originally rises were thought to be submissive signals, since female A. leptorhynchus commonly made them during social encounters. However, this assessment could have been an oversimplification. Females with high EODs tend to produce more GFRs when presented with EOD stimulus, while females with low EODs produce fewer. Conversely, the opposite is true in males (Dunlap and Oliveri, 2002; Zakon et al., 2002; Triefenbach and Zakon, 2003). It is believed that similarly to chirps, GFRs are emitted for different reasons in males and females. They do appear to be submissive signals in males, however, in females they appear to be more aggressive in nature. Often a lower EOD female will emit a long GFR in response to a female with a higher EOD (Zakon et al., 2002).

In contrast, AFRs are clearly aggressive signals and tend to be emitted during bouts of attacks in males and females equally. These signals consist of a series of repeated, brief increases in frequency, followed by gradual decreases in frequency back to baseline. Unlike chirps which are emitted at a distance from another fish and are believed to deter aggression, AFRs are emitted when fish are in close proximity (Engler and Zupanc, 2001; Hupé and Lewis, 2008) and thus signal imminent attacks by the emitter.

JAR occurs when two fish with similar EODs are in close proximity to one another. The two electrosensory systems "jam" each other because the low frequency AM beat interferes with the ability to locate low-frequency prey signals. To correct this situation one fish will shift its' EOD frequency away from the other, thus alleviating the "jam" (Lewis and Maler, 2002; Watanabe and Takeda, 1963). Some species, such as Eigenmannia, can either raise or lower their EOD. Conversely, A. leptorhynchus can only raise its EOD (Bullock et al., 1972; Heiligenberg et al., 1996; Metzner, 1999). JARs can last from seconds to hours and then the 
EOD will return to baseline. These are used when fish cannot move away from the other fish for some reason such as, fish are housed in community tanks or unable to leave their shelter during the day. When exposed to a jamming stimulus for an extended period of time, EOD circuitry shows forms of gradual EOD reset to the new baseline. This can be seen when the jamming stimulus is removed, but the higher EOD level is stable and maintained for hours. This is referred to as a long term frequency elevation (Zakon et al., 1999, 2002; Oestreich and Zakon, 2002). The intensity and duration of both JAR and long term frequency elevation are dependent upon the intensity and duration of the stimulus.

Chirps constitute their main communication signals. During a chirp there is an increase in EOD frequency of one fish that causes a transient increase in the frequency of the background $\mathrm{AM}$ beat of the combined signal from the two fish. It is this beat AM and the modulation of the beat that is perceived by the fish and encoded in its sensory system. Chirp structure (duration, frequency modulation peak and shape, etc.) varies across species. For some species, chirps come in distinct categories whereas in others, chirp properties vary along a continuum (Fig. 2). AFR and GFR also vary in duration and frequency across species but to a lesser degree (blue dots in Fig. 2). Our experiments focus, on three species of ghost knifefish that cover the range of variations in chirp properties observed in this family.

\section{Species-Specific Aspect of Electrocommunication}

The most studied species of ghost knifefish, Apteronotus leptorhynchus (brown ghost), is a moderately social species that displays much sexual dimorphism in body morphology and communication signals. EODs are sexually dimorphic with males discharging at higher frequencies $(800-1100 \mathrm{~Hz})$ than females $(600-800 \mathrm{~Hz})$. There is a linear correlation between size and EOD with larger more dominate male typically emitting the highest EOD (Tallarovic and Zakon, 2002). Female brown ghosts do not follow this same correlation (Dunlap et al., 1998; Smith, 2013). Males chirp at much higher rates than females (Dunlap et al., 1998), females are capable of producing chirps but rarely do so. Chirps are typically classified in 2 categories: small chirps and big chirps for this species. Both consist of a short increase in frequency accompanied by a linearly correlated decrease in amplitude. In all species of Apteronotids, the frequency rise is accompanied by a decrease in the amplitude of the EOD with the two parameters being 
linearly correlated. A. leptorhynchus small chirps are 10-40 ms long and 50-150 Hz in frequency increase whereas big chirps are 15-80 ms and 150-900 Hz (Dunlap et al., 1998; Bastian et al., 2001; Zakon et al., 2002; Hupé et al., 2008) (Fig. 2). The shape of the FM time course is reminiscent of a Gaussian curve. In A. leptorhynchus, chirps are emitted frequently in males near conspecific or when presented an EOD mimic of a male. When one fish chirps the other fish may answer within 500-1000 ms. This has been termed the echo response (Zupanc et al., 2006) and appears to be a submissive signal during aggressive interactions. If chirping is produced in a non-echo pattern, it can -on the contrary- signal the motivation to escalate the aggressive interaction (Hupé, 2012). Less is known about the use of chirps during courtship although it is clear that they play a role in courtship and in coordinating spawning. In this situation, males seem to chirp frequently but responses from the female also seem to be involved (Henninger, 2015). Since courtship behavior is typically not observed in captivity, the exact role of different types of chirps remains unclear.

Apteronotus albifrons (black ghost) is a solitary, aggressive species. EODs are sexually dimorphic with males discharging at lower frequencies than females (Hagedorn and Heiligenberg, 1985; Dunlap et al., 1998). Males tend to use more high frequency chirps than females (Allen and Marsat, 2019) as well as longer chirps. A. albifrons produce very few chirps in the conditions they have been submitted to in laboratory experiments (10 times less than $A$. leptorhynchus) and they do not appear to produce echo chirps. While chirps do not fall into distinct categories (Turner et al., 2007) they cover the same range of frequencies as $A$. leptorhynchus small and big chirps; their duration however is longer ranging from $50 \mathrm{~ms}$ to 500 ms. The shape of the FM time course is reminiscent of an $\alpha$-shape and in rare occasions can contain several peaks in frequency.

Adontosternarchus devenanzii (marble leaf knifefish) is the most social of the three species and the least studied. This species EOD discharges around 900-1200 Hz. A. devenanzii does not produce any low frequency chirps (Kolodziejski et al., 2007), instead it produces only high frequency (90-400 Hz of FM) long (50-500 ms) chirps similar to the high frequency chirps of A. leptorhynchus and A. albifrons. Chirps in this species tends to be more complex and variable, often possessing multiple frequency peaks (Fig. 2). A. albifrons can produce multi peaked chirps, however they are rare. Similarly, A. leptorhynchus is capable of producing very 
long duration chirps, but again these are rare. A. devenanzii also produces GFRs, which can be multi-peaked (Zhou and Smith, 2006). This has not been reported in either A. leptorhynchus or A. albifrons (Serrano-Fernandez, 2003; Triefenbach and Zakon, 2008). Current research thus suggests that $A$. devenanzii produce more diverse communication signals than the other two species considered here. There is no significant sexual dimorphism in body morphology or chirping (i.e. rate, use of peaks, and amount produced) in A. devenanzii (Zhou and Smith, 2006)

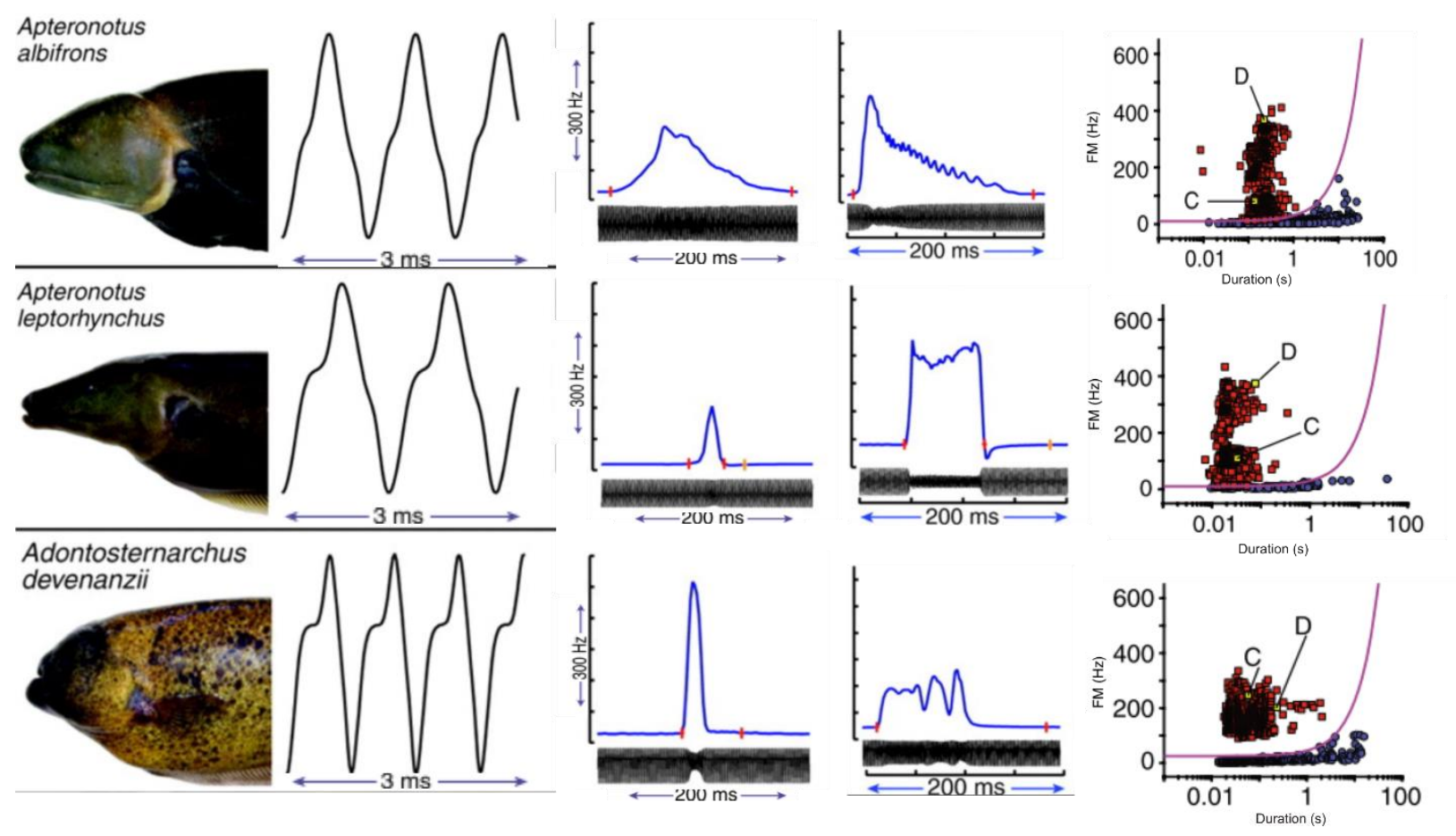

Figure 2. Properties of communication signals in 3 ghost knifefish. Figure modified from (Turner et al., 2007). To the far-left photographs of the species head. Second from the left, EOD traces showing differences in waveform. Third and fourth from the left, chirp examples showing the raw EOD trace (black) and the corresponding time course of the frequency increase (blue). The right most column is scatter plots of the frequency increase and duration of chirps (red) and AFRs/GFRs (blue). The purple line is the representation of the function that is used to distinguish chirps from AFRs/GFRs.

\section{Sensory Coding}

Neurophysiological experiments have pinpointed significant differences across the three species of ghost knifefish in the basic response properties of the neurons, thus suggesting that their sensory systems are adapted in different ways. In A. leptorhynchus, depending on the chirp type and the frequency of the background beat, chirps were encoded through one of two 
strategies (Fig. 3) in the primary sensory area known as the electrosensory lateral line lobe (ELL). Chirps occurring on a low frequency beat lead to synchronized bursting across the population of neurons, specifically pyramidal cells (PCs), allowing the accurate detection but not discrimination among chirps. Low-frequency beats are typical of same-sex aggressive interactions. Alternatively, chirps elicit graded heterogeneous responses allowing both detection and discrimination of the chirps when occurring on a high frequency beat (Allen and Marsat, 2018, 2019). Chirps on high frequency beats are typical of male-female interactions and occur during courtship.

A

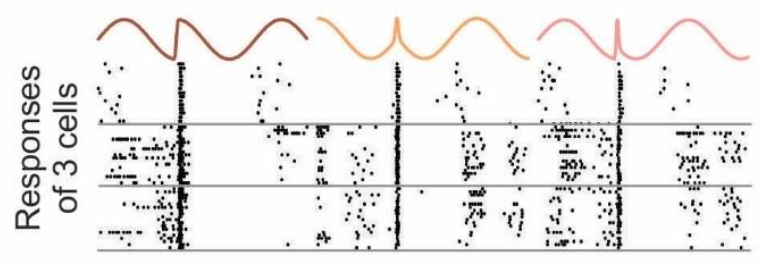

\section{C}

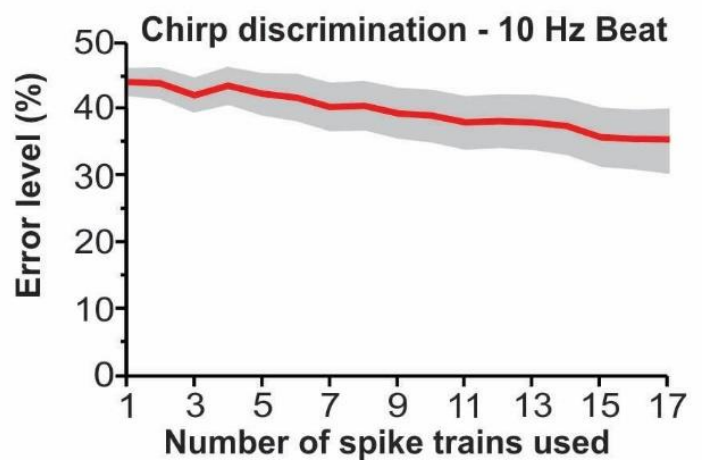

B
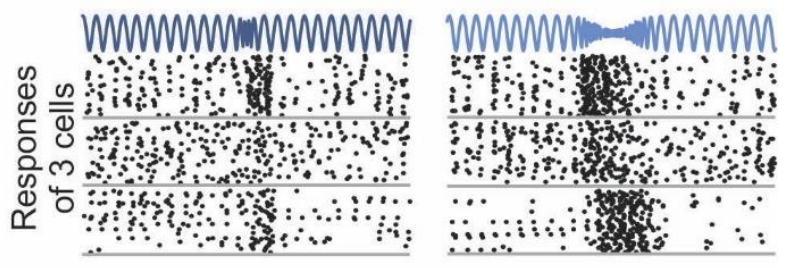

D

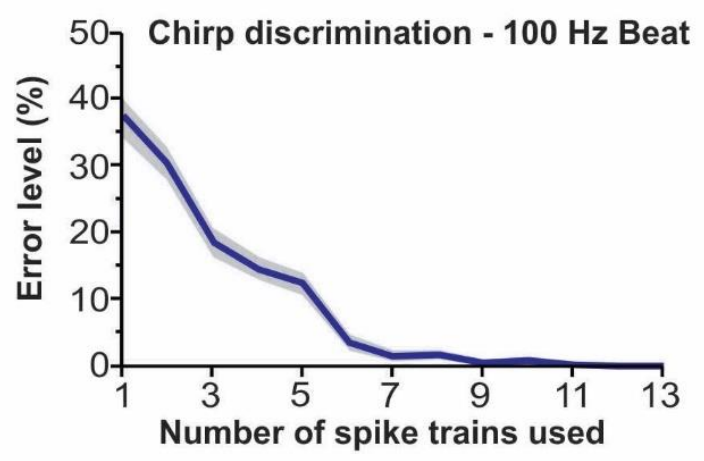

Figure 3. Neurophysiological responses of A. leptorhynchus ELL neurons showing that chirps on HFBs could be discriminated while responses for LFBs do not encode chirp properties. Modified from (Allen and Marsat, 2018). (A.) Responses from 3 PCs to chirps presented on a $10 \mathrm{~Hz}$ beat. The shape of the signal changes depending upon what phase the chirp occurs as shown by the fact that the first and second chirp from the left have the same properties (B.) Responses to chirps on $120 \mathrm{~Hz}$ beat. Note that for HFB phase is not a confounding factor and thus response pattern reflect chirp properties (e.g. duration). (C.) Analysis of response patterns quantifying the reliability with which chirps could be discriminated based on the information they contain as a function of the number of neurons included in the analysis. For LFB stimuli, even when pooling the information from many neurons, discrimination performance is near chance levels. (D.) Discrimination accuracy for chirps on HFBs reveal the high chirp encoding accuracy for these stimuli. 
Whereas A. leptorhynchus uses two coding strategies that are dependent upon the beat, $A$. albifrons appears to only use one coding strategy but the accuracy of encoding is context dependent. All chirps appear to be encoded via graded and heterogeneous increase or decreases in firing across PCs. (Allen and Marsat, 2019). Additionally, A. albifrons shows poor coding accuracy of chirps when the beat frequency is high $(100 \mathrm{~Hz})$, but better accuracy when the beat is low (10 Hz) (Fig. 4). Correspondingly, A. albifrons tends to chirp more when beat frequencies are low rather than high (Kolodziejski et al., 2007; Allen and Marsat, 2019). Despite these two species being closely related, there are differences in their chirps which may help to explain the differences in coding strategies. A. albifrons chirps are much longer than A. leptorhynchus. A. albifrons' chirps span more than one beat cycle, whereas A. leptorhynchus' chirps are short and do not span one beat cycle on LFBs. On HFBs the period becomes shorter and even small chirps will span more than one beat cycle (Walz et al., 2014; Allen and Marsat, 2019). Chirps that span less than a full cycle of the beat can be hard to discriminate because the shape of the signal is affected by both the chirp properties and the phase of the beat at which the chirp occurs. In $A$. leptorhynchus the coding strategy for LFB reflect this confounding factor and since it does not affect the longer A. albifrons chirps, an adapted coding strategy might not be required.
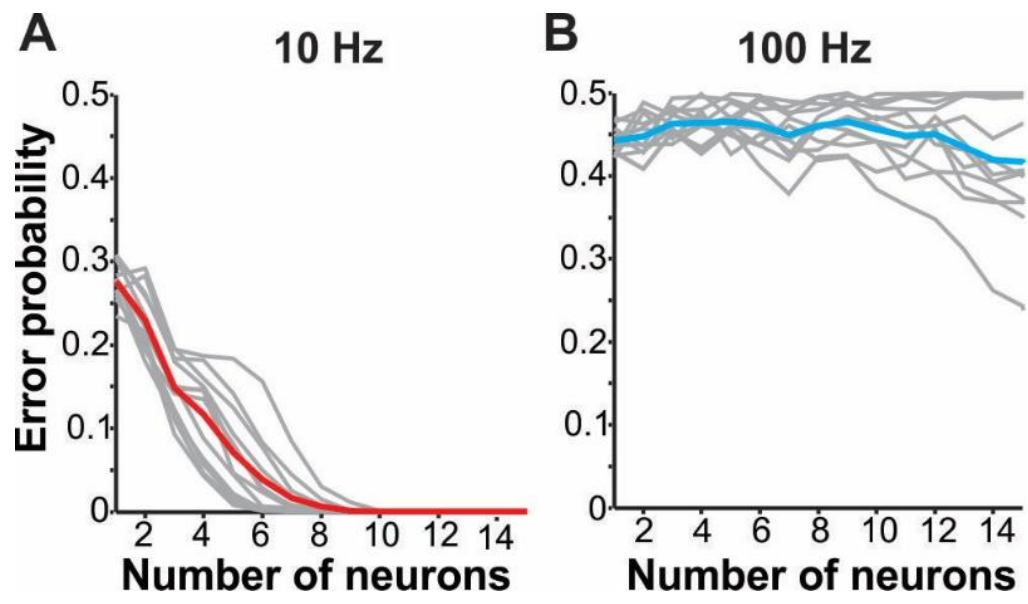

Figure 4. Neurophysiological responses of $A$. albifrons ELL neurons showing that chirps on LFBs could be discriminated while responses for HFBs do not encode chirp properties. Modified from (Allen and Marsat, 2019). (A.) Analysis of response patterns quantifying the reliability with which chirps could be discriminated based on the information they contain as a function of the number of neurons included in the analysis. For LFB stimuli, pooling the information from as little as 10 neurons would allow perfect discrimination of chirp properties. (B.) Discrimination accuracy for chirps on HFBs reveal poor encoding accuracy for these stimuli. 
A. devenanzii is the least studied of the three species. Being the most social and possessing the most complex chirps (often multi-peaked) it was thought to have the potential of displaying the highest level of discrimination. In a recent study (Allen, 2019) it was found that much like $A$. albifrons, $A$. devenanzii encoded chirps through graded, heterogeneous firing across PCs, with increased firing that corresponded with the FM and accompanied by the inversely correlated AM of the chirp. However, chirp coding was more accurate than for A. albifrons. This is due to the fact that $A$. devenanzii's PC responses did not adapt as much on HFB and thus details in the time course of the chirp could be seen in the PCs' firing rate. This was believed to suggest that $A$. devenanzii is capable of accurately coding -and thus discriminating- chirps on all frequencies of beat (Fig. 5).
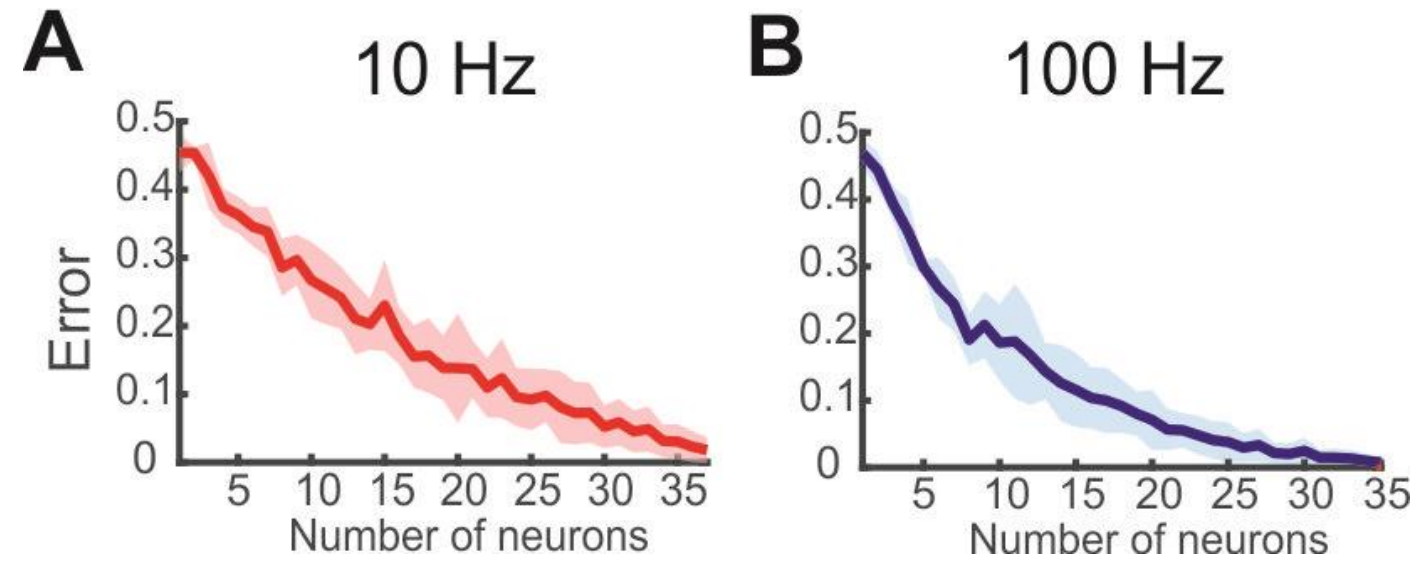

Figure 5. Neurophysiological responses of A. devenanzii ELL neurons showing the accurate discrimination of chirps for both LFBs and HFBs. Modified from (Allen, 2019). (A.) Analysis of response patterns quantifying the reliability with which chirps could be discriminated based on the information they contain as a function of the number of neurons included in the analysis. For LFB stimuli, pooling the information from a population of neurons would allow perfect discrimination of chirp properties. Note that the stimuli used in this experiment included noise whereas the results displayed in Fig 3-4 did not. (B.) Discrimination accuracy for chirps on HFBs also show the accurate encoding for these stimuli.

Neurophysiological results clearly suggest differences across the three species in the accuracy with which they could discriminate chirp properties with A. leptorhynchus being only capable of discriminating chirps on HFB, A. albifrons only on LFB and A. devenanzii being able to discriminate chirps on all beat frequencies. 
Here, we aim to confirm that the differences in sensory coding performance and strategies lead to differences in sensory discrimination behavior. We hypothesize that for the different signal types and species, the ability to discriminate small variations in chirp properties will match the neural encoding method and accuracy employed in the sensory system of each species. This hypothesis will be tested using a series of habituation-dishabituation assays in which changes in behavior, the result of perception and discrimination or the lack thereof, will be observed. We hope that this research will establish a clear link between signal structure, neural coding strategy and perceptual tasks. 


\section{Chapter 2: Methods}

\section{Fish Supplier and Housing}

Wild-caught Fish from the three focal species A. leptorhynchus, A. albifrons and A. devenanzii, were obtain from commercial suppliers and housed in $115 \mathrm{~L}$ tank. Water was maintained at $27^{\circ} \mathrm{C}$ and a conductivity of $200 \mu \mathrm{S}$. Individuals from each species were kept with 3-9 other conspecific if possible or were isolated for individual displaying aggressive bullying behavior. The tanks contain environmental enrichment items (tubes and/or plastic plants) and were kept on inverted light cycles so that their active phase happened when we conduct experiments. Sex and age of the individuals could not be determined from visual inspection, but none appeared to be juvenile. Fish were fed ad libitum and appeared to be in good health when they were used for experiments. Between 22 and 65 fish of each species were used and a given fish was never tested more often than once a day. We performed between 148 and 245 trials for each species

\section{Habituation-Dishabituation Paradigm}

The approach of this commonly used paradigm (Corfas and Dudai, 1989; Pinsker et al., 1970; Post and Vonderemde, 1999) is to habituate the animal to a specific stimulus that causes a behavioral response (e.g. swimming towards and around the stimulus source). After the long stimulation period with the first stimulus, the behavioral response decreased: it is habituated. Then a change in some parameter in the stimulus (e.g. chirp duration) is introduced. If a resurgence of the behavioral response is observed (i.e. dishabituation) it demonstrates that the animal was able to perceive the difference between the first and second stimulus. Following protocols that were previously established for these fish, we presented 1 min bouts of stimulation separated by 2 min pauses, this sequence of stimulation played for $90 \mathrm{~min}$ before the properties of the chirps was changed for another sequence of 4 stimulation bouts (Fig. 6, B). 

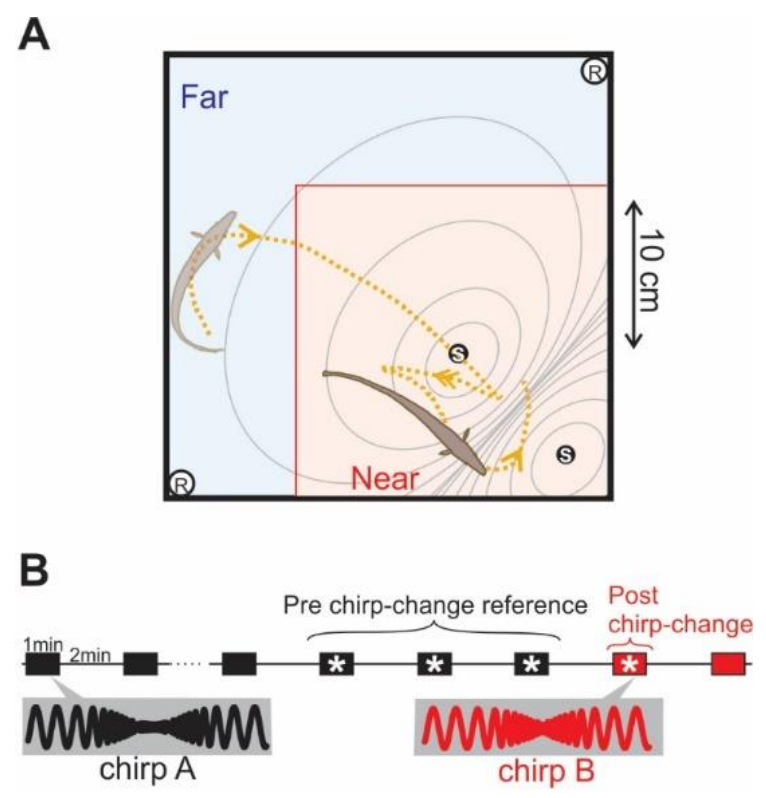

Figure 6. Habituation-Dishabituation testing set-up and analysis. Modified from (Allen and Marsat, 2018). Representation of the tanks (A.), where they are exposed to two stimuli. The first stimulus (chirp A) is played for approximately 90 minutes to habituate the fish. Then a second stimulus (chirp B) is played for approximately 15 minutes (B.). These stimuli are played for one minute and followed by a two-minute pause thought out the duration of the test. The stimuli are played from electrodes (S) and resulting signals from the fish (chirping) are recorded by recording electrodes (R). Infrared video recordings capture the fish's position throughout the experiment. (C.) An example swimming speed distribution during the habituation phase (black line) and the dishabituation phase (red line). The dashed line represents the median swimming speed of the three chosen stimulation points before the stimulus change. (D.) Example of the time spend swimming at fast speed vs. slow speed for during one experiment. The stars represent a fast swimming speed, while the circles represent a slower speed. The line represents the ratio of the two speeds and constitutes the speed index used to measure the strength of the behavioral response of the fish.

\section{Habituation-Dishabituation Tank Set-Up}

Fish were placed in an experimental tank that was roughly 30 x 30 centimeters wide with $10 \mathrm{~cm}$ of water (Fig. 6, A). The shallow depth restricted the movement to be mostly in 2 dimensions with limited up-and-down movement. The water was kept at similar conditions as the home tank. All tanks were shielded from light and video were captured in infrared light (Logitech C920, IR filter removed). Video was captured at 24 frames s$^{-1}$ with a spatial resolution of $1280 \times 720$ pixels. There were submerged stimulating dipoles that played the stimuli in one quadrant of the tank and a recording dipole placed perpendicularly to record the EOD output of 
the fish. The stimulus dipole shape (i.e. $10 \mathrm{~cm}$ apart) and signal strength mimics the average electric field of these fish. Additionally, the small size of this tank means the fish will receive a fairly strong and equal signal despite any changes in position. One to three fish were tested in parallel in adjacent tanks.

\section{Stimuli Parameters}

The fish were allowed to acclimate for twenty minutes before stimulation began. The fish's EOD frequency was determined, and the EOD frequency of the stimuli were set to cause a low frequency beat $(+10 \mathrm{~Hz}$ above the fish's own EOD frequency) or a high frequency beat $(+100 \mathrm{~Hz}$ or $+120 \mathrm{~Hz})$. Chirp properties were selected to encompass the range of chirp properties encountered naturally. This range was determined from previous publication and a publicly accessible database (hosted by the laboratory of G.T. Smith, Indiana University; http://www.indiana.edu/ efishlab/catalog/faqs.php). Pairs of chirps with properties at opposite ends of the spectrum observed were chosen. Different set of stimuli were tested to focus on a specific chirp category (e.g. small chirps), a specific aspect of the signal (i.e. number of frequency peaks) or to have stimuli as different as possible within the range of natural chirps. Chirp pairs as tested on a given beat frequency (low or high) which was always the same for the first and second stimuli in the pair. The relevant aspects of the chirps and the stimuli are detailed in Table 1 and include number of frequency peaks, chirp width (ms), chirp height $(\mathrm{Hz})$, and the beat frequency. The shape of the chirp FM replicated natural chirps: chirps for A. leptorhynchus were a Gaussian curve FM, while A. albifrons stimuli were $\alpha$-shaped FM, and A. devenanzii were more complexly shaped FM. The shape of the EOD cycle itself in the artificial stimulus realistically replicated the species-specific shape of each species' EOD rather than simply using sinusoidal sinewaves as was often done in previous research. 
Table 1. Stimuli Sets for A. devenanzii, A. albifrons, and A. leptorhynchus. Each stimuli set is tailored for the individual species. Each set contains two stimuli. Stimulus 1 (Chirp A in Fig. 2) is played during the first 90 minutes during the habituation period. Then stimulus 2 (Chirp B in Fig. 6) is played for approximately 15 minutes. It is during this time the fish will dishabituate if it can detect differences between the stimuli.

\begin{tabular}{|c|c|c|c|c|c|}
\hline \multicolumn{2}{|c|}{ Adontosternarchus devenanzii } & \multirow{2}{*}{$\begin{array}{c}\begin{array}{c}\text { Number } \\
\text { of Peaks }\end{array} \\
2 \\
\end{array}$} & \multirow{2}{*}{$\begin{array}{c}\begin{array}{c}\text { Chirp Width } \\
\text { (ms) }\end{array} \\
100 \\
\end{array}$} & \multirow{2}{*}{$\begin{array}{c}\begin{array}{c}\text { Chirp } \\
\text { Height } \\
(\mathbf{H z})\end{array} \\
100 \\
\end{array}$} & \multirow{2}{*}{$\begin{array}{c}\begin{array}{c}\text { EOD } \\
\text { Frequency } \\
\text { (Hz re. } \\
\text { focal fish) }\end{array} \\
100\end{array}$} \\
\hline Set 1 & Stimuli 1 & & & & \\
\hline & Stimuli 2 & 8 & 200 & 400 & 100 \\
\hline \multirow[t]{2}{*}{ Set 2} & Stimuli 1 & 8 & 200 & 400 & 100 \\
\hline & Stimuli 2 & 2 & 100 & 100 & 100 \\
\hline \multirow[t]{2}{*}{ Set 3} & Stimuli 1 & 1 & 40 & 100 & 10 \\
\hline & Stimuli 2 & 1 & 100 & 250 & 10 \\
\hline \multirow[t]{2}{*}{ Set 4} & Stimuli 1 & 1 & 100 & 250 & 10 \\
\hline & Stimuli 2 & 1 & 40 & 100 & 10 \\
\hline \multirow[t]{2}{*}{ Set 5} & Stimuli 1 & 1 & 40 & 100 & 100 \\
\hline & Stimuli 2 & 1 & 100 & 250 & 100 \\
\hline \multirow[t]{2}{*}{ Set 6} & Stimuli 1 & 1 & 100 & 250 & 100 \\
\hline & Stimuli 2 & 1 & 40 & 100 & 100 \\
\hline \multicolumn{2}{|c|}{ Apteronotus albifrons } & $\begin{array}{l}\text { Number } \\
\text { of Peaks }\end{array}$ & $\begin{array}{l}\text { Chirp Width } \\
\text { (ms) }\end{array}$ & $\begin{array}{c}\text { Chirp } \\
\text { Height } \\
(\mathbf{H z})\end{array}$ & $\begin{array}{c}\text { EOD } \\
\text { Frequency } \\
\text { (Hz re. } \\
\text { focal fish) } \\
\end{array}$ \\
\hline \multirow[t]{2}{*}{ Set 1} & Stimuli 1 & 1 & 100 & 200 & 100 \\
\hline & Stimuli 2 & 1 & 200 & 350 & 100 \\
\hline \multirow[t]{2}{*}{ Set 2} & Stimuli 1 & 1 & 100 & 50 & 100 \\
\hline & Stimuli 2 & 1 & 200 & 150 & 100 \\
\hline \multirow[t]{2}{*}{ Set 3} & Stimuli 1 & 1 & 50 & 50 & 100 \\
\hline & Stimuli 2 & 2 & 200 & 350 & 100 \\
\hline \multirow[t]{2}{*}{ Set 4} & Stimuli 1 & 1 & 50 & 50 & 10 \\
\hline & Stimuli 2 & 1 & 50 & 100 & 10 \\
\hline \multirow[t]{2}{*}{ Set 5} & Stimuli 1 & 1 & 100 & 200 & 10 \\
\hline & Stimuli 2 & 1 & 200 & 350 & 10 \\
\hline \multirow[t]{2}{*}{ Set 6} & Stimuli 1 & 1 & 50 & 50 & 10 \\
\hline & Stimuli 2 & 1 & 200 & 350 & 10 \\
\hline \multicolumn{2}{|c|}{ Apteronotus leptorhynchus } & $\begin{array}{l}\text { Number } \\
\text { of Peaks }\end{array}$ & $\begin{array}{l}\text { Chirp Width } \\
\text { (ms) }\end{array}$ & $\begin{array}{c}\text { Chirp } \\
\text { Height } \\
\text { (Hz) }\end{array}$ & $\begin{array}{c}\text { EOD } \\
\text { Frequency } \\
\text { (Hz re. } \\
\text { focal fish) } \\
\end{array}$ \\
\hline \multirow[t]{2}{*}{ Set 1} & Stimuli 1 & 1 & 10 & 60 & 10 \\
\hline & Stimuli 2 & 1 & 15 & 122 & 10 \\
\hline \multirow[t]{2}{*}{ Set 2} & Stimuli 1 & 1 & 10 & 60 & 120 \\
\hline & Stimuli 2 & 1 & 15 & 122 & 120 \\
\hline \multirow[t]{2}{*}{ Set 3} & Stimuli 1 & 1 & 15 & 122 & 10 \\
\hline & Stimuli 2 & 1 & 10 & 60 & 10 \\
\hline \multirow[t]{2}{*}{ Set 4} & Stimuli 1 & 1 & 15 & 300 & 120 \\
\hline & Stimuli 2 & 1 & 45 & 900 & 120 \\
\hline
\end{tabular}




\section{Data Analysis}

Although pilot data sets relied on the fish's EOD response or the quantification of visually-identified lunging behavior to access fish responsiveness, the data presented in the result focused on the swimming behavior recorded and quantified from video recordings. The video data collected from each bout of 1 min stimulation was imported to MATLAB and a custom program tracked the movement of the fish. The semi-autonomous program tracked the head, tail, and 2D center of mass (Fig. 7). Changes in position and speed were calculated from the frame, time, and spatial calibration during the stimulation period. Accuracy of the results was verified visually and frames with incorrect tracking were corrected manually. In pilot studies, we found that the best indicator of response strength was a normalized swimming speed measure. This speed index was calculated by first determining a threshold speed to divide swimming movements into fast and slow (Fig. 6, C). For each analysis, this threshold was taken as the median swimming speed for the three last stimuli of the habituation phase of the assay. The index is then calculated for each stimulation bout as the ratio of time spent at high speed over time spent at low speed (Fig. 6, D). The index will therefore be a average 1 by definition for the last three point before the dishabituating stimuli is played. We also quantified the distance between the "2D center of mass" of the fish's image and the center of the stimulus dipole and we plot the average distance for each stimulation bout. All data satisfied the assumptions of normality (as assessed with Kolmogorov-Smirnov tests) and thus we used either ANOVAs or paired t-test to test the significance of our results.
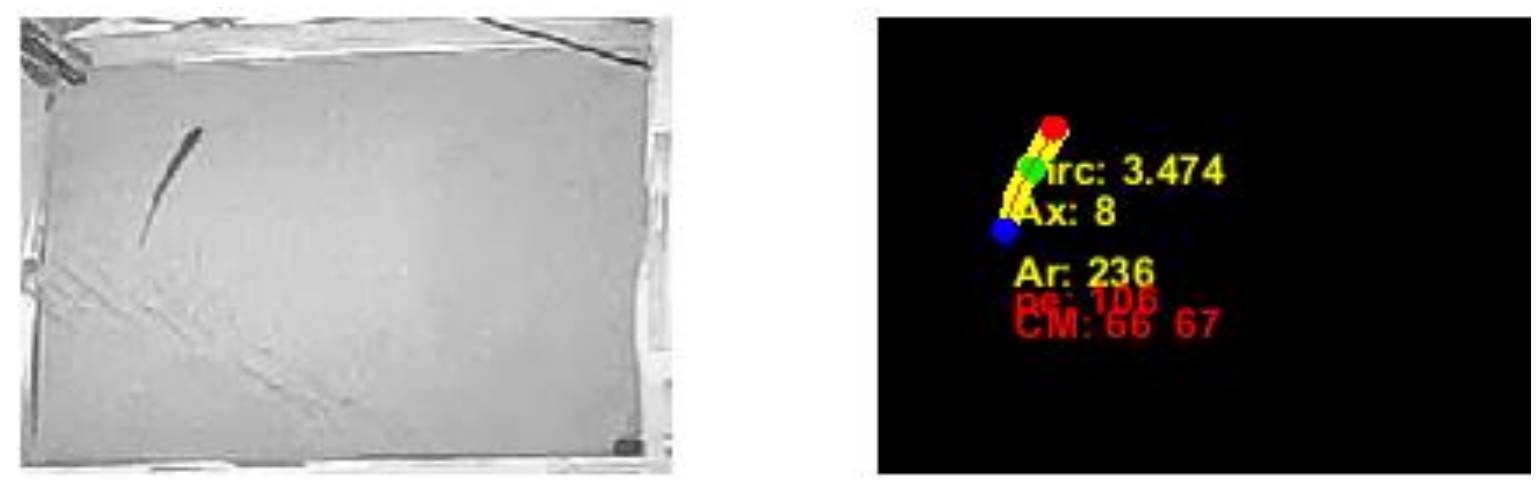

Figure 7 Custom tracking program. This semi-autonomous program calculated for each frame of the video (e.g. panel on the left) the position of the head of the fish (i.e. wider end of the fish's shadow), the tail, and 2D center of mass (e.g. results displayed on the right panel). These values are saved for later analysis. 


\section{Chapter 3: Results}

The fish's reaction to communication signals has several components. For most species, the most marked component is a change in locomotor activity relative to the stimulus source. As in natural interaction, a fish presented with a mimic of conspecific signals will start swimming faster around the signal source as a way to investigate the stimulus. It will often lunge and produce attack movements directed at the location of the stimulus source. The fish can also spend more time near the stimulus electrode or produce chirps in response to the stimulus. The reaction to these signals habituate with repeated presentation but the response can regain strength when a novel stimulus is presented. Therefore, in these fish as in many other species, a habituation-dishabituation test can be used to test whether the animal discriminates between two signals.

In a pilot project we examined which aspect of the behavior displayed the most reliable habituation in different species. To do so we used a beat stimulus (no chirp) that changed frequency to elicit a dishabituation response because it has been shown to be an aspect of conspecific signals that fish strongly react to. In some species (e.g. A. leptorhynchus) lunging or chirping displayed a habituation-dishabituation effect, but in all species, we found the swimming patterns to have the most marked habituation/dishabituation. The results presented here thus consist of the analysis of the movement patterns of these fish. Since fast swimming towards and around the stimulation electrodes is characteristic of the response, we quantified swimming speed and the position of the fish relative to the electrode. We normalized swimming speed for each fish relative to the fish's median speed during the habituated portion of the test and expressed the result as a speed index representing the ratio of fast over slow speeds (see methods for details).

\section{A. leptorhynchus}

In A. leptorhynchus, the fish have a strong initial reaction, swimming 2-3 times more often at high speed than low speed (Fig. 8 A-B). We tested discrimination of chirp properties by 

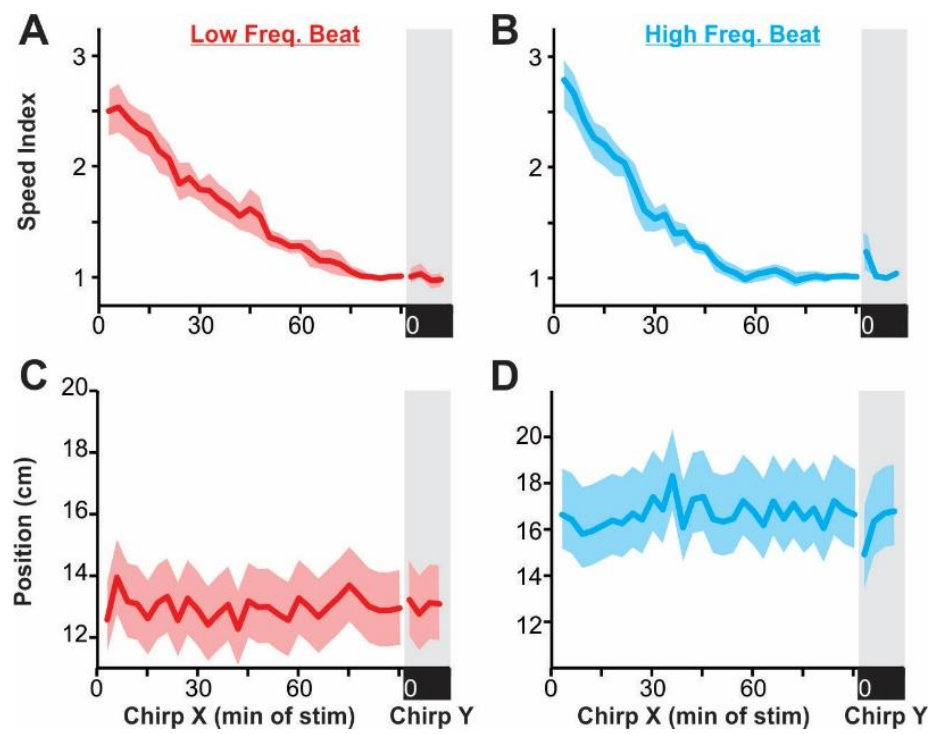

Figure 9. A. leptorhynchus shows strong dishabituation for HFBs chirp stimuli when considering only the first $5 \mathrm{~s}$ of each stimulation bout. (A) The effect of chirps on swimming speed adjusted to the calculated swim-speed index are shown over time for the first $5 \mathrm{~s}$ of stimulus. (B) The same is displayed for chirps on high-frequency beats (C-D) The position relative to the stimulating electrode is plotted for the first $5 \mathrm{~s}$ of each stimulation bout.
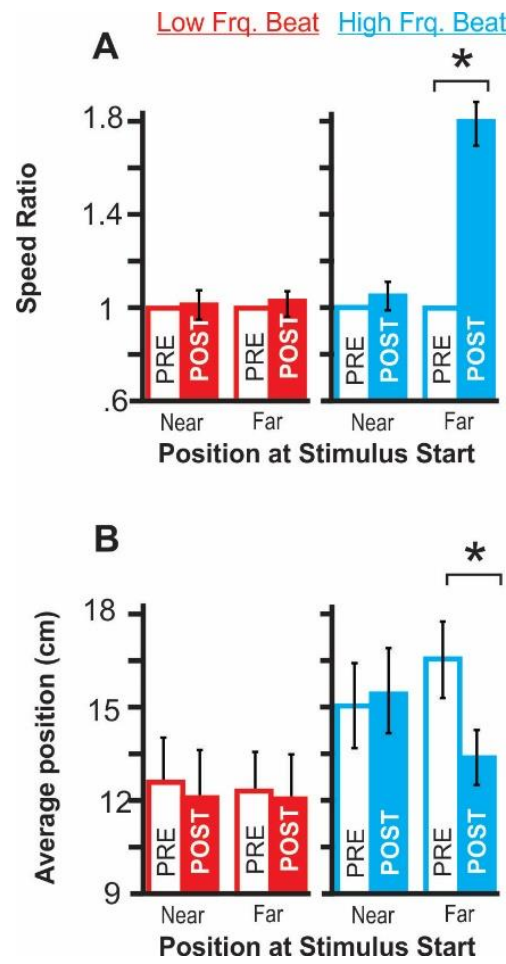

Figure 8. A. leptorhynchus responses to chirp on HFBs or LFBs measured over the 1 min of each stimulation bout. (A) The effect of chirps on swimming speed adjusted to the calculated swim-speed index are shown over time for the first $1 \mathrm{~min}$ of stimulus during the habituation portion (chirp X) or after chirp properties are changed (chirp Y). (B) The same is represented for chirps on high-frequency beats (C-D) The average distance relative to the stimulating electrode is calculated for each $1 \mathrm{~min}$ stimulation bout. Each panel show the average \pm s.e. for 4 stimuli pairs and 80 (LFBs) or 165 (HFBs) trials.
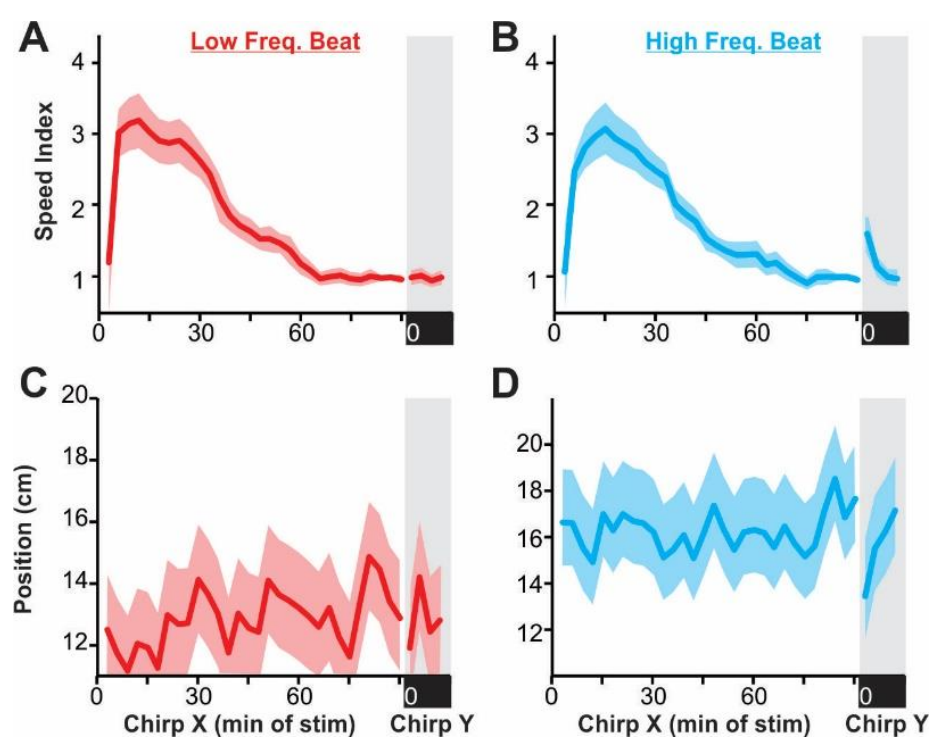

Figure 10. A. leptorhynchus responses displayed a dishabituation effect only if the fish is far from the stimulus when a new chirp is introduced. Trials where divided based on the position of the fish just before the new stimulus (post) started playing: either near the stimulation electrode or far from the stimulation electrode (see Fig. 6, A) Average speed (A) and position (B) was calculated for the first $5 \mathrm{~s}$ of the habituated stimulation bouts (last three pre-stimulus change) or for the first $5 \mathrm{~s}$ of the stimulus with changed chirp properties (post: filled bars). Stars indicate paired t-test results of $\mathrm{p}<0.01$. 
varying the duration and/or frequency change of the chirps between the first (habituating stimulus; marked "Chirp X" in figures) and second stimulus (dishabituating stimulus; "Chirp Y") as shown in Table 1. Using a variety of chirp pairs, we found no effects of chirp properties on the strength of the dishabituation effect (data not shown), thus we pooled together stimuli sets that had the same background beat. As explained in the introduction, the background beat is a quasi-sinusoidal AM resulting from the interaction of two fish's EODs and is thus always present when two fish interact. In sexually dimorphic fish, low frequency beats (LFBs) typically occur during same-sex interactions while high frequency beats (HFBs) are typical of male-female interactions. We found that the speed index did not increase when a novel chirp stimulus is played after the habituating phase for LFB stimuli (ANOVA followed by Tukey HSD for the last 3 points of the habituating phase compared to the first novel stimulation; $0.37<\mathrm{P}<0.43$ ). Chirps played on HFBs showed a small and not significant increase in swimming activity after the chirp change (ANOVA followed by Tukey HSD; $0.12<\mathrm{P}<0.23$ ).

We observed that fish sometimes reacted more strongly in the first seconds of the 1 minlong bouts of stimulation. We therefore examined the habituation-dishabituation effect based on only the first $5 \mathrm{~s}$ of stimulation of each 1 min bout. The dishabituation effect was stronger when considering the initial reaction to each stimulus (Fig. 9) and significantly different for the novel chirp compared to the habituated response when the beat is HFB (ANOVA followed by Tukey HSD; $0.02<\mathrm{P}<0.04)$. We noticed that this dishabituation effect was strong in some trials but hardly noticeable in others and we determined that the distance to the electrode when the stimulus started seemed to correlate with the presence or absence of a dishabituation effect. When the fish was far from the electrode when a new HFB stimulus started, it approached the electrode and swimming speed clearly increased (Fig. 10, A; paired t-test, $\mathrm{P}<0.01$ ). This resurgence of rapid movement was not observed when the fish is close to the stimulation electrode at the start of the stimulus or for LFB stimuli (paired t-test, $\mathrm{P}>0.1$ ).

This tendency to investigate new stimuli from a distant source leads to a change in average position relative to the stimulus. When these fish initially react to the habituating stimulus they perform large movement to and from the stimulus location and thus their average distance to the electrode is no larger than when -in the habituated state- its movement are less 

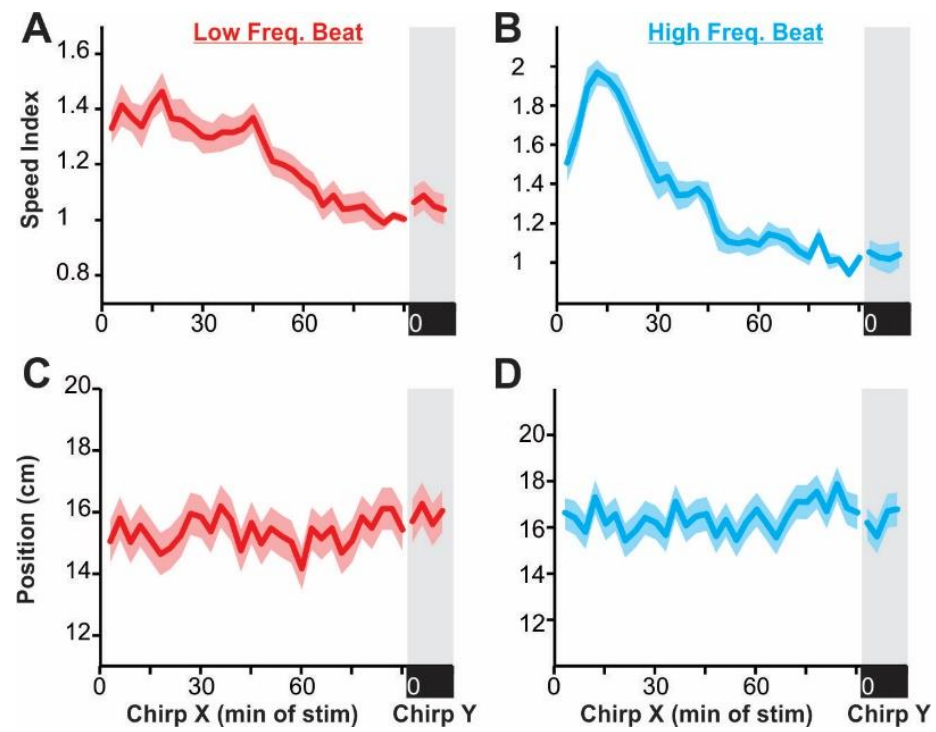

Figure 11. A. albifrons responses to chirp on HFBs or LFBs measured over the 1 min of each stimulation bout. (A) The effect of chirps on swimming speed adjusted to the calculated swim-speed index are shown over time for the first 1 min of stimulus during the habituation portion (chirp X) or after chirp properties are changed (chirp Y). (B) The same is represented for chirps on high-frequency beats (C-D) The average distance relative to the stimulating electrode is calculated for each $1 \mathrm{~min}$ stimulation bout. Each panel show the average \pm s.e. for 6 stimuli pairs and 66 (LFBs) or 82 (HFBs) trials.

Figure 12. A. albifrons shows dishabituation for LFBs chirp stimuli when considering only the first $5 \mathrm{~s}$ of each stimulation bout. (A) The effect of chirps on swimming speed adjusted to the calculated swim-speed index are shown over time for the first $5 \mathrm{~s}$ of stimulus. (B) The same is displayed for chirps on high-frequency beats $(\mathbf{C}$ D) The position relative to the stimulating electrode is plotted for the first $5 \mathrm{~s}$ of each stimulation bout.
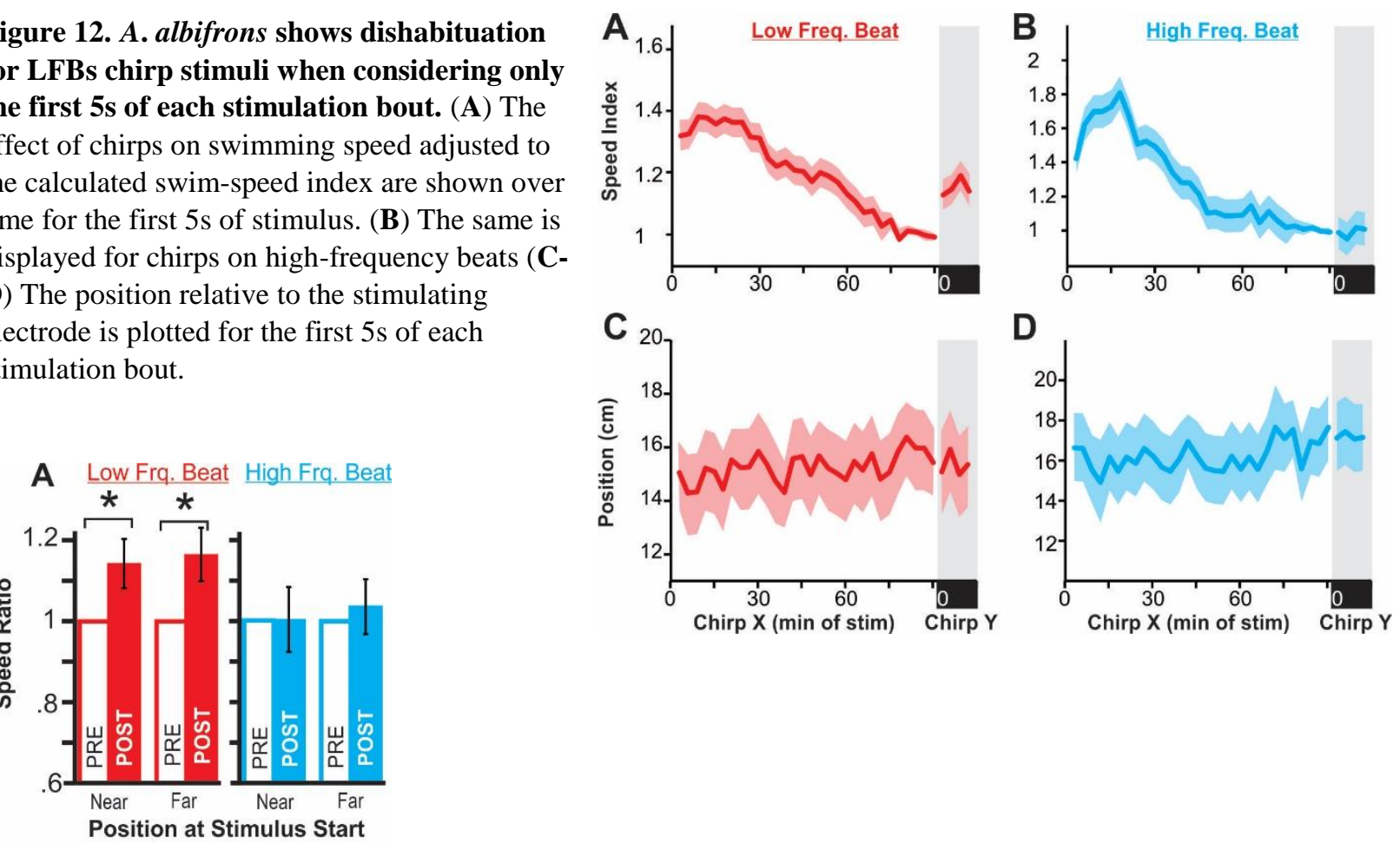

B

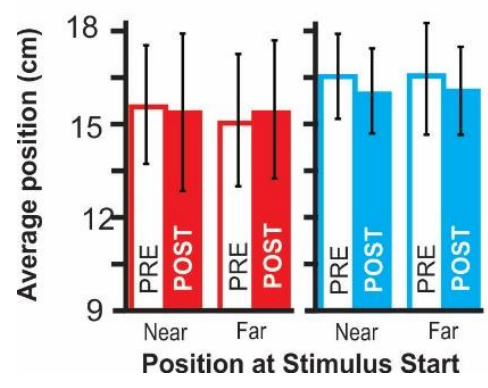

Figure 13. A. albifrons shows an increase in swimming speed for LFB after chirp change regardless of the position of the fish at the start of the stimulus. Trials where divided based on the position of the fish just before the new stimulus (post) started playing: either near the stimulation electrode or far from the stimulation electrode (see Fig. 6, A) Average speed (A) and position (B) was calculated for the first $5 \mathrm{~s}$ of the habituated stimulation bouts (last three pre-stimulus change) or for the first $5 \mathrm{~s}$ of the stimulus with changed chirp properties (post: filled bars). Stars indicate paired t-test results of $\mathrm{p}<0.01$. 
specifically directed at the electrode. A dishabituating stimulus starting at a distance brings the fish towards the stimulus location and its movements are more tightly centered on the signal's origin. As a result, average distance from the stimulus does not vary much through stimulus repetitions (Fig. 8-9, C-D) except for a dishabituating pulse, on a HFB, starting when the fish was far from the electrode (Fig. 10, B paired t-test, $\mathrm{P}>0.1$ ). In this circumstance, the fish swam closer to the electrodes for a few seconds.

\section{A. albifrons}

The behavior of $A$. albifrons is in many ways similar to that of A. leptorhynchus with a few critically important differences. First the habituation of the reaction to chirp stimuli is less pronounced leading to smaller differences in swimming speeds between the initial and habituated responses. Most importantly the relationship between the dishabituation effect and the beat frequency was inversed in A. albifrons. Dishabituation is clear only for LFBs and the difference between habituated speed index, and speed in response to the new stimulus is significant when considering the first $5 \mathrm{~s}$ of each stimuli only (Fig. 11-12 A). Furthermore, the rebound in response is significantly higher than habituated baseline not just for the first stimulus after the change in chirp properties but for the 4 stimulations plotted on the figures (ANOVA followed by Tukey HSD; $0.003<\mathrm{P}<0.01)$. Dishabituation effect for HFB stimuli or for the full $1 \mathrm{~min}$ response to LFB is not significant (ANOVA followed by Tukey HSD for the last 3 points of the habituating phase compared to the first novel stimulation; $0.23<\mathrm{P}<0.62$ ).

Position of the fish relative to the stimulation dipole also did not relate to the behavior in the same way as observed in A. leptorhynchus. Here, there is no increase in approaching the signal source that resulted in the fish being on average closer to the electrode (ANOVA followed by Tukey HSD; $0.31<\mathrm{P}<0.47$ ) before vs. after the stimulus change. Also, the position of the fish at the start of the dishabituating stimulus (near to vs far from the stimulation dipole) does not influence the dishabituation effect; there is a significant increase in swimming speed in both cases for LFB (Fig. 13A; pair t-test, $\mathrm{P}=0.004$ and $\mathrm{P}=0.002$ ) for near and far respectively. There is no significant change as chirp is altered for speed in response to HFB (pair t-test, $\mathrm{P}=0.6$ and $\mathrm{P}=0.55$ for near and far respectively) or for position (Figure 13B; pair t-test, $\mathrm{P}>0.1$ ). These 

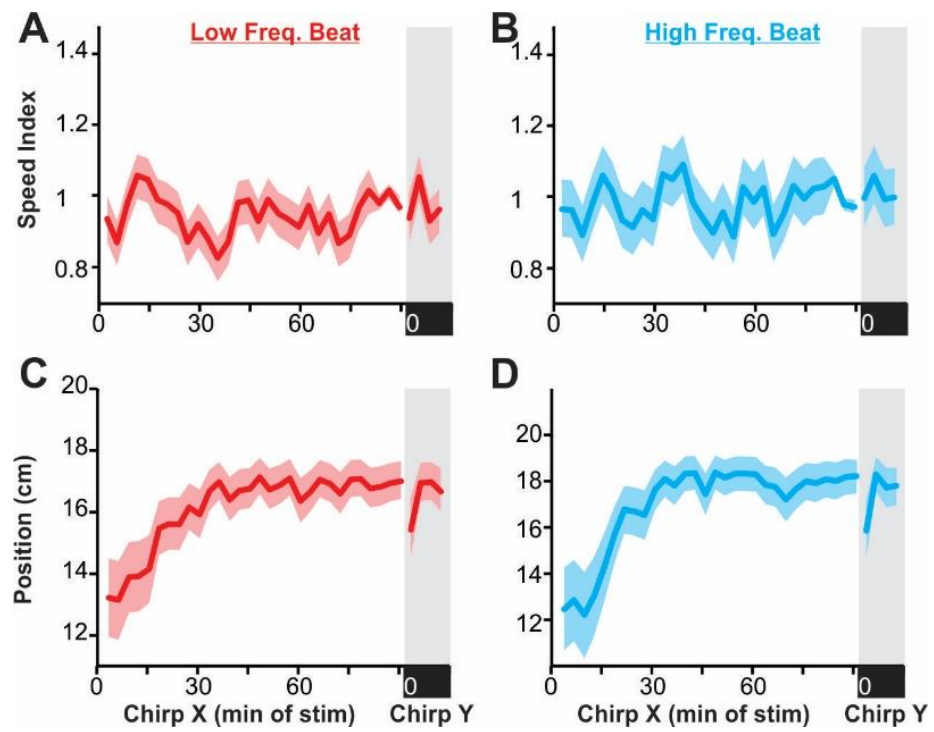

Figure 15 A. devenanzii response during the first $5 \mathrm{~s}$ of stimulus show dishabituation for all chirp stimuli when considering the first $5 \mathrm{~s}$ of each stimulation bout. (A) The effect of chirps on swimming speed adjusted to the calculated swim-speed index are shown over time for the first 5 s of stimulus. (B) The same is displayed for chirps on high-frequency beats (C-D) The position relative to the stimulating electrode is plotted for the first $5 \mathrm{~s}$ of each stimulation bout.

\section{A Low Frq. Beat High Frq. Beat}
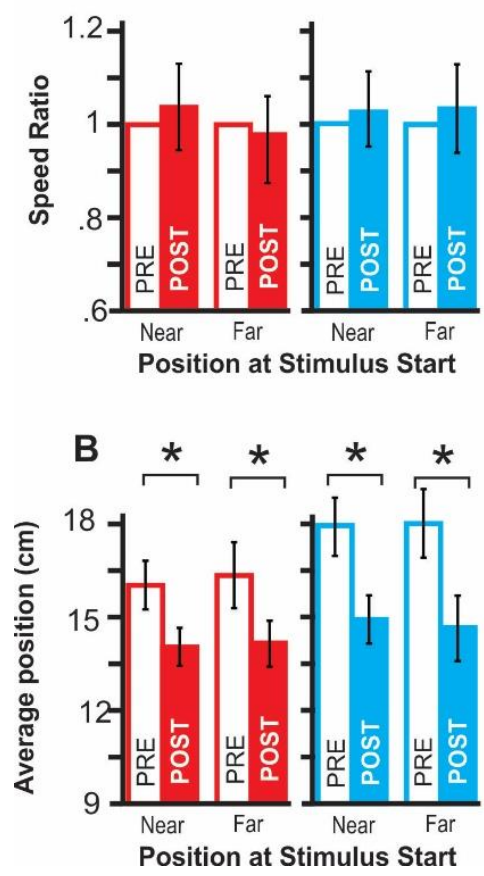

Figure 14. A. devenanzii responses to chirp on HFBs or LFBs measured over the 1 min of each stimulation bout. (A) The effect of chirps on swimming speed adjusted to the calculated swim-speed index are shown over time for the first 1 min of stimulus during the habituation portion (chirp X) or after chirp properties are changed (chirp Y). (B) The same is represented for chirps on high-frequency beats (C-D) The average distance relative to the stimulating electrode is calculated for each $1 \mathrm{~min}$ stimulation bout. Each panel show the average \pm s.e. for 6 stimuli pairs and 63 (LFBs) or 179 (HFBs) trials.
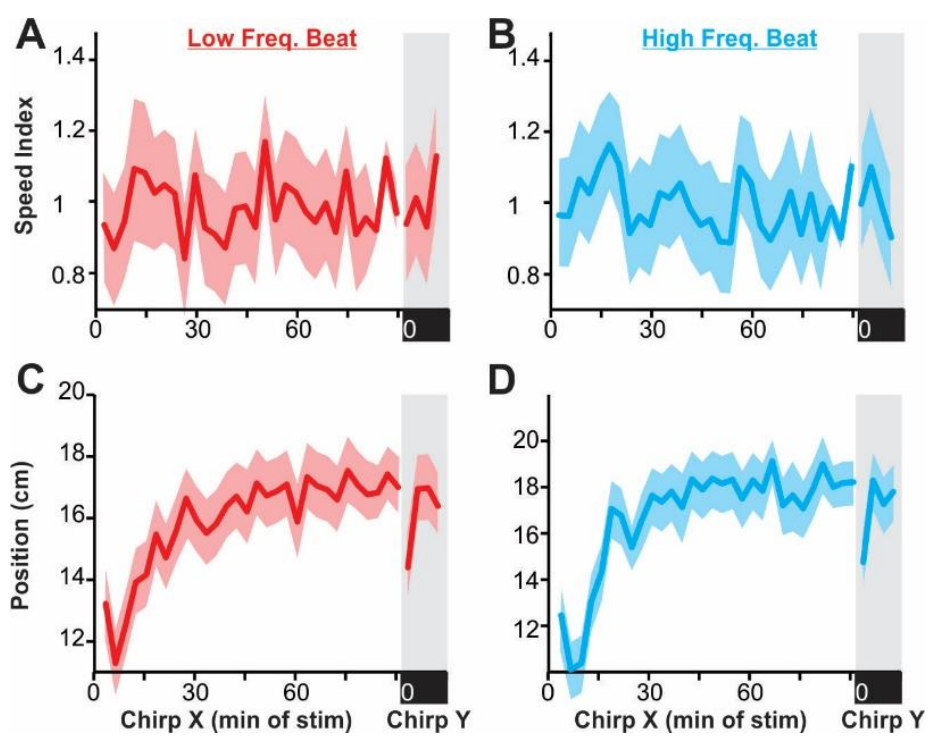

Figure 16 A. devenanzii shows dishabituation in position not speed regardless of stimulus type or position when the stimulus starts. Trials where divided based on the position of the fish just before the new stimulus (post) started playing: either near the stimulation electrode or far from the stimulation electrode (see Fig, 6, A) Average speed (A) and position (B) was calculated for the first $5 \mathrm{~s}$ of the habituated stimulation bouts (last three prestimulus change) or for the first $5 \mathrm{~s}$ of the stimulus with changed chirp properties (post: filled bars). Stars indicate paired t-test results of $\mathrm{p}<0.01$. 
differences suggest species-specificity in motor patterns for the various behavioral effects (see discussion).

\section{A. devenanzii}

The last species examined also presented species-specific effects in the behavioral patterns observed in response to the habituation-dishabituation test. The most striking particularity of $A$. devenanzii is that swimming speed does not change over the course of the repeated stimulation (Fig. 14-15, panels A-B). As a result, no dishabituation effect is observed with the swimming speed, independently of the beat frequency or the position of the fish just prior to the start of the novel stimulus for both the whole $1 \mathrm{~min}$ and first $5 \mathrm{~s}$ of the stimulation bout (Fig. 16 A; paired t-test, P>0.1).

In contrast with the behavior patterns of A. leptorhynchus and A. albifrons, we observed in this species a clear habituation in the position of the fish relative to the stimulus dipole (Fig. 14-15, panels C-D). The fish were, in average, several centimeters closer to the electrode at the beginning of the habituation period. Then towards the end of the habituation period, the fish was several centimeters further from the electrode (habituation), reflecting the fact that it spent a larger portion of the time close to the signal source. Most importantly, in all stimulation paradigms, we observed a dishabituation effect for both LFBs and HFBs and whether or not we consider only the first 5s (ANOVA followed by Tukey HSD for the last 3 points of the habituating phase compared to the first novel stimulation; $0.001<\mathrm{P}<0.04$ ). As with A. albifrons, position at the start of the new stimulus had no influence on the significance of the dishabituation effect which was absent when considering speed (Fig. 16A; paired t-tests; P>0.1) and significant when considering position (Fig. 16, B; paired t-tests; $\mathrm{P}<0.05$ ). 


\section{Chapter 4: Discussion}

Our habituation-dishabituation assay reveals differences across the three species. In both A. leptorhynchus and A. albifrons, dishabituation is observed only for one range of beat frequency. HFBs allowed chirp properties to be discriminated by A. leptorhynchus and LFBs lead to a dishabituation response in A. albifrons. For A. devenanzii, beat frequency did not influence the dishabituation effect and discrimination ability was observed for all beat frequencies and all chirp pairs tested. Furthermore, the three species presented subtle differences in their behavioral response. For example, changes in reactivity to the stimulus was observed mostly in the swimming speed of the individuals for A. leptorhynchus and A. albifrons but in the location of the fish relative to the stimulus for A. devenanzii. Also, behavior was affected by the distance to the stimulation electrode at the start of new -dishabituating-stimuli for $A$. leptorhynchus but not the other two species. While these additional differences simply emphasize the fact that communication and social behaviors varies across species, differences in dishabituation effect clearly speaks to the differences in sensory functions and how different chirp stimuli are discriminated.

\section{Sensory Processing and Sender-Receiver Matching}

The discrimination ability displayed in the habituation-dishabituation assay closely matched the sensory coding performance of PC neurons in the primary sensory area. Specifically, A. leptorhynchus showed accurate coding of chirp properties for HFBs but not for LFB (Marsat et al., 2012; Allen and Marsat, 2018). LFB stimuli elicited a stereotyped burst response that cannot encode adequately the chirp structure but that efficiently signals the occurrence of the chirp. HFBs however elicit graded and heterogeneous responses in PC neurons that accurately encode chirp structure. We showed here that these two coding strategies are matched with the corresponding discrimination performance behaviorally.

In A. albifrons there is also a difference in coding accuracy depending of the frequency of the beat but only one coding strategy is used: graded heterogeneous responses (Allen and Marsat, 2019). HFB stimuli elicited noisy responses that adapted quickly after the start of the chirp 
whereas responses to LFB stimuli accurately represented chirp structure. As a consequence, the analysis of neural responses leads to the prediction that chirps on HFBs would not be discriminated accurately but that LFB chirps could. Our behavioral analysis supports this conclusion.

A. devenanzii were also tested neurophysiologically to determine their coding accuracy for chirp stimuli (Allen, 2019). The ELL responses accurately encoded the chirp's structure in graded population responses for all beat frequencies. It was concluded that the information to discriminate among chirps was accurately relayed to higher brain areas and could influence behavior. By showing that these fish dishabituated to the chirp stimuli when chirp properties are changed, we demonstrated that they indeed perceived differences in chirp properties for all the stimuli we tested.

The match between sensory coding and behavioral performance suggest that the speciesspecific adaptation in neurophysiology match the behavioral output displayed by the fish. Our results thus support the idea that adaptations in sensory coding strategies and encoding accuracy accompany changes in communication and sensory behaviors.

\section{Limitations of Our Behavioral Analysis}

An obvious limitation of our behavioral assay is that, like almost all animal behavioral paradigm, we are not testing directly the perceptual abilities of the animal. It is possible that the animal was capable of perceptually distinguishing between the habituating and dishabituating stimuli, but this perceived difference did not elicit a behavioral reaction. While this fact limits the conclusions, we can draw from a negative result (lack of dishabituation effect) we argue that the differences we observed are still meaningful. Although the information might have been present at a perceptual level, the fact is that in some cases it led to a behavioral output and in some cases it did not. Therefore, our experiments demonstrate that there is a difference in how relevant the information is to the behavior even though we cannot claim that the difference originated from a perceptual difference. Furthermore, our conclusions are also supported by differences in sensory coding (Allen and Marsat, 2018, 2019; Allen, 2019) that also suggest that different amount of information reaches the higher brain areas. Together, the neurophysiological 
data previously published (Marsat and Maler, 2010) and the behavioral results presented here clearly reveal qualitative differences in discrimination of chirps.

The behavioral test performed here represent a limited range of situations compared to the full range of behaviorally relevant interaction that these fish can experience. Particularly, in a courtship situation, physiological and behavioral changes unique to this context could influence how sensory signals are processed and perceived (Hagehorn and Heiligenberg, 1985; Silva et al., 2008). For example, we know that serotonin can affect both the chirping behavior and the way chirps are encoded in the ELL (Telgkamp et al., 2007; Deemyad et al., 2013). It is thus possible that in different context, chirps that failed to be discriminated in our experiment would be discriminated during courtship. This factor might explain why A. albifrons did not appear to distinguish chirp properties when presented on high-frequency beats typical of male-female interactions. Behavioral tests aimed at detailing the importance of chirp's properties during courtship are an obvious next step to improve our understanding of the communication behavior in weakly electric fish.

\section{Ethological Relevance of Sensory Functions.}

The differences revealed in our experiments could be explained by differences in behavioral tasks performed by each species and their ethological particularities. A striking difference between $A$. devenanzii and the other two species is that it is the only one that does not display sexual dimorphism in EOD frequency (Zhou and Smith, 2006). The frequency of the beat (i.e. LFB vs HFB) is thus not indicative of whether the interaction is between same sex individuals or not. Since the interaction between same sex individuals is often different from interaction between males and females, it could explain why A. leptorhynchus and A. albifrons display different discrimination behavior based on beat frequency but not A. devenanzii.

Previous studies (Marsat and Allen, 2019) show that A. albifrons rarely chirp during interactions with HFBs - at least outside of courtship. This suggest that there is less of a need to finely discriminate between chirp's variants in this context and would explain the poor chirp coding for HFB stimuli and the lack of behavioral discrimination in our experiments. 
A. leptorhynchus frequently exchange chirps during male-male interactions (Engler et al., 2000; Hupé and Lewis, 2008) even though the neurophysiology and the behavior indicate a lack of discrimination of chirp properties. This counter-intuitive result can easily be explained by the fact that A. leptorhynchus chirps are short and span less than one cycle of a low frequency beat. For this reason, a given chirp will cause a variety of AM in the signal reaching the receiver depending on the phase of the beat at which it starts. Since chirp phase cannot be controlled by the sender (Walz et al., 2013), chirp properties are not easily extracted from the signal being perceived. Correspondingly the nervous system uses a neural code that is optimized for detection -not discrimination- and the behavior is not influenced by chirp properties for these LFBs (Allen and Marsat, 2018). We therefore argue that there is a match between signal structure, coding strategy and behavioral performance. This species exchange chirp back and forth during agonistic interaction (i.e. typically low frequency beats) (Hupé, 2012). We speculate that, if the pattern of chirp exchange carries information there might not be a need to rely of chirp properties to mediate the interaction, only chirp rate and timing.

The three species also display ethological differences in the size of their social group: $A$. devenanzii are more social and are often found in groups of several individuals (Zhou and Smith, 2006), whereas A. albifrons are clearly more territorial and solitary to the point that many individuals do not tolerate tank mates in the lab. A. leptorhynchus do not cluster in large groups but are often found with a few other conspecifics (Stamper et al., 2010). In a range of species, higher sociality and social complexity is correlated with a richer communication repertoire. The social complexity theory stipulates that communication signals must be more diverse in species with more complex social structure to mediate the wider range of social situations. Our analysis would require a wider sampling of species in this branch of gymnotiforms and should involve a characterization of signals complexity to directly support this theory. Nevertheless, it can be useful to view our results in the context of this theory. A. devenanzii have very variable chirp structure and are more social than the other two species. It is possible that accurate perception of chirp properties is particularly important in this species to support the rich social interactions they experience. 


\section{Conclusions}

Our comparative analysis of sensory-driven behavior reveals differences in the communication system of three species of weakly electric fish. Informative aspects of communication signals should be encoded accurately and used by the organism to guide behavior. We suggest that our result point to differences in which aspect of the signals are informative in the different species and consequently, the sensitivity of the nervous system and the perceptual abilities vary correspondingly. Our research thus improves our understanding of how communication signal, sensory physiology and behavioral tasks co-evolve and are matched to one another. 


\section{References}

Allen K (2019) Neural Adaptations for Communication in Three Species of Weakly Electric FIsh. PhD Thesis, West Virginia Univ.

Allen KM, Marsat G (2018) Task-specific sensory coding strategies are matched to detection and discrimination performance. J Exp Biol 221:jeb170563.

Allen KM, Marsat G (2019) Neural Processing of Communication Signals: The Extent of Sender-Receiver Matching Varies across Species of Apteronotus. eneuro 6:ENEURO.039218.2019.

Alves-Gomes A, Orti F-G, Haygood M, Heiligenberg W (1995) Phylogenetic analysis of the South American electric fishes (order Gymnotiformes) and the evolution of their electrogenic system: a synthesis based on morphology, electrophysiology, and mitochondrial sequence data. Mol Biol Evol.

Alves-Gomes J (2001) The evolution of electroreception and bioelectrogenesis in teleost fish: a phylogenetic perspective. J Fish Biol 58:1489-1511.

Baker CA, Ma L, Casareale CR, Carlson BA (2016) Behavioral and Single-Neuron Sensitivity to Millisecond Variations in Temporally Patterned Communication Signals. J Neurosci 36:8985-9000.

Bastian J, Nguyenkim J (2001) Dendritic modulation of burst-like firing in sensory neurons. J Neurophysiol 85:10-22.

Bastian J, Schniederjan S, Nguyenkim J (2001) Arginine vasotocin modulates a sexually dimorphic communication behavior in the weakly electric fish Apteronotus leptorhynchus. J Exp Biol 204:1909-1923.

Bradbury JW, Vehrencamp SL (2011) Principles of Animal Communication, Second Edition literatury. In: Principles of Animal Communication, 2nd ed.

Buck J, Buck E (1966) Biology of Synchronous Flashing of Fireflies. Nature 211:562-564.

Bullock TH, Hamstra RH, Scheich H (1972) The jamming avoidance response of high frequency electric fish - II. Quantitative aspects. J Comp Physiol 77:23-48.

Clutton-Brock TH, Albon SD (1979) The Roaring of Red Deer and the Evolution of Honest Advertisement. Behaviour 69:145-170.

Compton W, Albert J (2006) CommunicationinFishes. Commun fishes 2:647-731.

Corfas G, Dudai Y (1989) Habituation and dishabituation of a cleaning reflex in normal and mutant Drosophila. J Neurosci 9:56-62.

Cuddy M, Aubin-Horth N, Krahe R (2012) Electrocommunication behaviour and non invasivelymeasured androgen changes following induced seasonal breeding in the weakly electric fish, Apteronotus leptorhynchus. Horm Behav 61:4-11. 
David Smith JL, McDougal C, Miquelle D (1989) Scent marking in free-ranging tigers, Panthera tigris. Anim Behav 37:11-27.

Dawkins MS, Guilford T (1991) The corruption of honest signalling. Anim Behav 41:865-873.

Deemyad T, Metzen MG, Pan Y, Chacron MJ (2013) Serotonin selectively enhances perception and sensory neural responses to stimuli generated by same-sex conspecifics. Proc Natl Acad Sci U S A 110:19609-19614.

Dunlap KD, Oliveri LM (2002) Retreat site selection and social organization in captive electric fish, Apteronotus leptorhynchus. J Comp Physiol A Neuroethol Sensory, Neural, Behav Physiol 188:469-477.

Dunlap KD, Thomas P, Zakon HH (1998) Diversity of sexual dimorphism in electrocommunication signals and its androgen regulation in a genus of electric fish, Apteronotus. J Comp Physiol - A Sensory, Neural, Behav Physiol 183:77-86.

Engler G, Fogarty CM, Banks JR, Zupanc GKH (2000) Spontaneous modulations of the electric organ discharge in the weakly electric fish, Apteronotus leptorhynchus: A biophysical and behavioral analysis. J Comp Physiol - A Sensory, Neural, Behav Physiol.

Engler G, Zupanc G (2001) Differential production of chirping behavior evoked by electrical stimulation of the weakly electric fish, Apteronotus leptorhynchus. J Comp Physiol - A Sensory, Neural, Behav Physiol 187:747-756.

Gall MD, Brierley LE, Lucas JR (2012) The sender-receiver matching hypothesis: support from the peripheral coding of acoustic features in songbirds. J Exp Biol 215:3742-3751.

Guilford T, Dawkins M (1993) Receiver Psychology and the Design of Animal Signals. Trends Neurosci 16:430-436.

Hagedorn M, Heiligenberg W (1985) Court and spark: electric signals in the courtship and mating of gymnotoid fish. Anim Behav 33:254-265.

Hasson O (1994) Cheating Signals. J Theor Biol 167:223-238.

Heiligenberg W, Wong CJH, Metzner W, Keller CH (1996) Motor control of the jamming avoidance response of Apteronotus leptorhynchus: evolutionary changes of a behavior and its neuronal substrates. J Comp Physiol A 179:653-674.

Henninger J (2015) Social Interactions in Natural Populations of Weakly Electric Fish.

Hopkins CD (1976) Stimulus filtering and electroreception: Tuberous electroreceptors in three species of Gymnotoid fish. J Comp Physiol a A.

Hopkins CD (1981) Neuroethology of Electric Communication. Trends Neurosci 4:4-6.

Hupé GJ (2012) Electrocommunication in a species of weakly electric fish, Apteronotus leptorhynchus: Signal patterning and behaviour.

Hupé GJ, Lewis JE (2008) Electrocommunication signals in free swimming brown ghost knifefish, Apteronotus leptorhynchus. J Exp Biol 211:1657-1667.

Hupé GJ, Lewis JE, Benda J (2008) The effect of difference frequency on 
electrocommunication: chirp production and encoding in a species of weakly electric fish, Apteronotus leptorhynchus. J Physiol Paris 102:164-172.

Kolodziejski JA, Sanford SE, Smith GT (2007) Stimulus frequency differentially affects chirping in two species of weakly electric fish: implications for the evolution of signal structure and function. J Exp Biol 210:2501-2509.

Kostarakos K, Hedwig B (2012) Calling song recognition in female crickets: Temporal tuning of identified brain neurons matches behavior. J Neurosci 32:9601-9612.

Kupfermann I, Castellucci V, Pinsker H, Kandel E (1970) Neuronal correlates of habituation and dishabituation of the gill-withdrawal reflex in Aplysia. Science (80- ) 167:1743-1745.

Lewis JE, Maler L (2002) Dynamics of Electrosensory Feedback: Short-Term Plasticity and Inhibition in a Parallel Fiber Pathway.

Loyau A, Saint Jalme M, Cagniant C, Sorci G (2005) Multiple sexual advertisements honestly reflect health status in peacocks (Pavo cristatus). Behav Ecol Sociobiol 58:552-557.

Marsat G, Longtin A, Maler L (2012) Cellular and circuit properties supporting different sensory coding strategies in electric fish and other systems. Curr Opin Neurobiol.

Marsat G, Maler L (2010) Neural Heterogeneity and Efficient Population Codes for Communication Signals. J Neurophysiol 104:2543-2555.

Martínez Wells M, Henry CS (1992) Behavioural responses of green lacewings (Neuroptera: Chrysopidae: Chrysoperla) to synthetic mating songs. Anim Behav 44:641-652.

Metzner W (1999) Neural circuitry for communication and jamming avoidance in gymnotiform electric fish. J Exp Biol 202:1365-1375.

Moran G (1984) Vigilance Behaviour and Alarm Calls in a Captive Group of Meerkats, Suricata suricatta. Z Tierpsychol 65:228-240.

Oestreich J, Zakon HH (2002) The long-term resetting of a brainstem pacemaker nucleus by synaptic input: A model for sensorimotor adaptation. J Neurosci 22:8287-8296.

Post N, von der Emde G (1999) The "novelty response" in an electric fishresponse properties and habituation. Physiol Behav 68:115-128.

Richards DG (1981) Alerting And Message Components In Songs Of Rufous-Sided Towhees. Behaviour 76:223-249.

Rodd FH, Hughes KA, Grether GF, Baril CT (2002) A possible non-sexual origin of mate preference: Are male guppies mimicking fruit? Proc R Soc B Biol Sci 269:475-481.

Ryan M, Wilczynski W (2019) Coevolution of Sender and Receiver : Effect on Local Mate Preference in Cricket Frogs Author ( s ): Michael J . Ryan and Walter Wilczynski Published by: American Association for the Advancement of Science Stable.

Salazar VL, Krahe R, Lewis JE (2013) The energetics of electric organ discharge generation in gymnotiform weakly electric fish. J Exp Biol 216.

Serrano-Fern $>$ ndez P (2003) Gradual frequency rises in interacting black ghost knifefish, 
Apteronotus albifrons. J Comp Physiol A Sensory, Neural, Behav Physiol 189:685-692.

Silva A, Quintana L, Perrone R, Sierra F (2008) Sexual and seasonal plasticity in the emission of social electric signals. Behavioral approach and neural bases. J Physiol Paris 102:272-278.

Smith GT (2013) Evolution and hormonal regulation of sex differences in the electrocommunication behavior of ghost knifefishes (Apteronotidae). J Exp Biol 216:24212433.

Stamper SA, Carrera-G E, Tan EW, Fugère V, Krahe R, Fortune ES (2010) Species differences in group size and electrosensory interference in weakly electric fishes: Implications for electrosensory processing. Behav Brain Res 207:368-376.

Tagliacollo VA, Bernt MJ, Craig JM, Oliveira C, Albert JS (2016) Model-based total evidence phylogeny of Neotropical electric knifefishes (Teleostei, Gymnotiformes). Mol Phylogenet Evol 95:20-33.

Tallarovic SK, Zakon HH (2002) Electrocommunication signals in female brown ghost electric knifefish, Apteronotus leptorhynchus. J Comp Physiol A Neuroethol Sens Neural Behav Physiol 188:649-657.

Tallarovic SK, Zakon HH (2005) Electric organ discharge frequency jamming during social interactions in brown ghost knifefish, Apteronotus leptorhynchus. Anim Behav 70:13551365.

Telgkamp P, Combs N, Smith GT (2007) Serotonin in a diencephalic nucleus controlling communication in an electric fish: Sexual dimorphism and relationship to indicators of dominance. Dev Neurobiol 67:339-354.

Triefenbach F, Zakon H (2003) Effects of sex, sensitivity and status on cue recognition in the weakly electric fish Apteronotus leptorhynchus. Anim Behav 65:19-28.

Triefenbach FA, Zakon HH (2008) Changes in signalling during agonistic interactions between male weakly electric knifefish, Apteronotus leptorhynchus. Anim Behav 75:1263-1272.

Turner CR, Derylo M, de Santana CD, Alves-Gomes JA, Smith GT (2007) Phylogenetic comparative analysis of electric communication signals in ghost knifefishes (Gymnotiformes: Apteronotidae). J Exp Biol 210:4104-4122.

Walz H, Grewe J, Benda J (2014) Static frequency tuning accounts for changes in neural synchrony evoked by transient communication signals. J Neurophysiol 112:752-765.

Walz H, Hupé GJ, Benda J, Lewis JE (2013) The neuroethology of electrocommunication: How signal background influences sensory encoding and behaviour in Apteronotus leptorhynchus. J Physiol 107:13-25.

Watanabe A, Takeda K (1963) The Change of Discharge Frequency by A.C. Stimulus in a Weak Electric Fish. J Exp Biol 40:57-66.

Wilczynski W, McClelland BE, Rand AS (1993) Acoustic, auditory, and morphological divergence in three species of neotropical frog. J Comp Physiol A 172:425-438.

Wollerman L, Wiley RH (2002) Background noise from a natural chorus alters female 
discrimination of male calls in a neotropical frog. Anim Behav 63:15-22.

Woolley SMN, Moore JM (2011) Coevolution in communication senders and receivers: vocal behavior and auditory processing in multiple songbird species. Ann N Y Acad Sci 1225:155-165.

Zakon H, Oestreich J, Tallarovic S, Triefenbach F (2002) EOD modulations of brown ghost electric fish: JARs, chirps, rises, and dips. J Physiol Paris 96:451-458.

Zakon HH, Smith GT (2010) Weakly electric fish: Behavior, neurobiology, and neuroendocrinology. In: Hormones, Brain and Behavior Online, pp 611-639.

Zakon, Mcanelly, Smith, Dunlap, Lopreato, Oestreich, Few (1999) Plasticity of the electric organ discharge: implications for the regulation of ionic currents. J Exp Biol 202:1409-1416.

Zhou M, Smith GT (2006a) Structure and sexual dimorphism of the electrocommunication signals of the weakly electric fish, Adontosternarchus devenanzii. J Exp Biol 209:48094818.

Zupanc GKH, Sîrbulescu RF, Nichols A, Ilies I (2006) Electric interactions through chirping behavior in the weakly electric fish, Apteronotus leptorhynchus. J Comp Physiol A Neuroethol Sensory, Neural, Behav Physiol 192:159-173. 